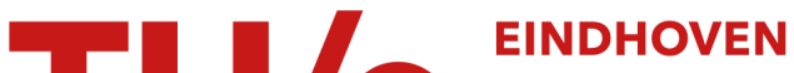 \\ UNIVERSITY OF \\ TECHNOLOGY
}

\section{Microscopically derived free energy of dislocations}

\author{
Citation for published version (APA): \\ Kooiman, M., Hütter, M., \& Geers, M. G. D. (2015). Microscopically derived free energy of dislocations. Journal \\ of the Mechanics and Physics of Solids, 78, 186-209. https://doi.org/10.1016/j.jmps.2015.02.007
}

DOI:

10.1016/j.jmps.2015.02.007

Document status and date:

Published: 01/01/2015

\section{Document Version:}

Publisher's PDF, also known as Version of Record (includes final page, issue and volume numbers)

\section{Please check the document version of this publication:}

- A submitted manuscript is the version of the article upon submission and before peer-review. There can be important differences between the submitted version and the official published version of record. People interested in the research are advised to contact the author for the final version of the publication, or visit the $\mathrm{DOI}$ to the publisher's website.

- The final author version and the galley proof are versions of the publication after peer review.

- The final published version features the final layout of the paper including the volume, issue and page numbers.

Link to publication

\section{General rights}

Copyright and moral rights for the publications made accessible in the public portal are retained by the authors and/or other copyright owners and it is a condition of accessing publications that users recognise and abide by the legal requirements associated with these rights.

- Users may download and print one copy of any publication from the public portal for the purpose of private study or research.

- You may not further distribute the material or use it for any profit-making activity or commercial gain

- You may freely distribute the URL identifying the publication in the public portal.

If the publication is distributed under the terms of Article $25 \mathrm{fa}$ of the Dutch Copyright Act, indicated by the "Taverne" license above, please follow below link for the End User Agreement:

www.tue.nl/taverne

Take down policy

If you believe that this document breaches copyright please contact us at:

openaccess@tue.nl

providing details and we will investigate your claim. 


\title{
Microscopically derived free energy of dislocations
}

\author{
M. Kooiman ${ }^{\text {a }}$, M. Hütter ${ }^{\text {b,* }}$, M.G.D. Geers ${ }^{a}$ \\ ${ }^{a}$ Eindhoven University of Technology, Mechanics of Materials, Department of Mechanical Engineering, PO Box 513,5600 MB Eindhoven, \\ The Netherlands \\ ${ }^{\mathrm{b}}$ Eindhoven University of Technology, Polymer Technology, Department of Mechanical Engineering, PO Box 513, 5600 MB Eindhoven, \\ The Netherlands
}

\section{A R T I C L E I N F O}

\section{Article history:}

Received 15 July 2014

Received in revised form

16 December 2014

Accepted 9 February 2015

Available online 11 February 2015

Keywords:

Dislocations

Microstructures

Crystal plasticity

Metallic materials

Coarse graining

\begin{abstract}
A B S T R A C T
The dynamics of large amounts of dislocations is the governing mechanism in metal plasticity. The free energy of a continuous dislocation density profile plays a crucial role in the description of the dynamics of dislocations, as free energy derivatives act as the driving forces of dislocation dynamics.

In this contribution, an explicit expression for the free energy of straight and parallel dislocations with different Burgers vectors is derived. The free energy is determined using systematic coarse-graining techniques from statistical mechanics. The starting point of the derivation is the grand-canonical partition function derived in an earlier work, in which we accounted for the finite system size, discrete glide planes and multiple slip systems. In this paper, the explicit free energy functional of the dislocation density is calculated and has, to the best of our knowledge, not been derived before in the present form.

The free energy consists of a mean-field elastic contribution and a local defect energy, that can be split into a statistical and a many-body contribution. These depend on the density of positive and negative dislocations on each slip system separately, instead of GND-based quantities only. Consequently, a crystal plasticity model based on the here obtained free energy, should account for both statistically stored and geometrically necessary dislocations.
\end{abstract}

(c) 2015 Elsevier Ltd. All rights reserved.

\section{Introduction}

The governing mechanism of metal plasticity is the dynamics of dislocations, which are line-like defects in the crystal structure. Crystals can contain up to $10^{9}$ dislocation lines intersecting a square millimeter. Therefore, the collective behavior of many dislocations together determines the mechanical properties associated with crystal plasticity.

A number of dynamical frameworks have been developed to describe the dynamics of dislocation densities, in which the free energy plays a key role, see e.g. Groma (1997), Gurtin (2000), Gurtin and Anand (2005), Gurtin et al. (2007) and Gurtin (2008, 2010). Moreover, stationary states have been derived from the free energy, see e.g.. Groma et al. (2006), Scardia et al. (2014), and Geers et al. (2013).

To obtain the equilibrium behavior and driving forces for dislocations on a macroscopic scale, it is thus necessary to have a free energy expression that results from coarse-graining the microscopic description of dislocations. Furthermore, a derivation from the microscopic level could help in choosing proper macroscopic variables for a dynamical model.

\footnotetext{
* Corresponding author.

E-mail addresses: M.Kooiman@tue.nl (M. Kooiman), M.Huetter@tue.nl (M. Hütter), M.G.D.Geers@tue.nl (M.G.D. Geers).
} 
In the literature, several attempts have been made to retrieve the free energy of dislocations. First, different phenomenological assumptions are made to match different macroscopic plasticity models, see e.g. Ertürk et al. (2009), Bayley et al. (2007), Svendsen (2002), Klusemann et al. (2012) and Bargmann and Svendsen (2012). These free energy expressions are all local or weakly non-local in terms of the dislocation densities.

Second, straight dislocations were considered as an example of two-dimensional Coulomb particles that interact with a logarithmic interaction potential, see e.g. the work of Kosterlitz an Thouless (Kosterlitz and Thouless, 1973; Nelson, 1978; Nelson and Halperin, 1979), Mizushima (1960), Ninomiya (1978) and Yamamoto and Izuyama (1988). In these papers, the free energy of systems with an homogeneous dislocation density was derived. This system exhibits a dislocation mediated melting transition. Below the critical temperature, dislocations occur in tightly bound pairs, but above this temperature, dislocation pairs tend to unbind, and thereby destroy the long-range order in a two-dimensional crystal. However, the anisotropic character of the dislocation interaction was not taken into account in these works, and the effect of mechanical loading was not considered.

Third, the free energy of dislocations was derived by Groma and coworkers using a mean-field assumption in the coarsegraining (Groma and Balogh, 1999; Groma et al., 2006, 2007). As the physical temperature of the system is almost zero relative to the other characteristic energy scales at hand, a second, phenomenological temperature is introduced to obtain a non-vanishing statistical contribution, which results in screening.

Fourth, the equilibrium dislocation profile of a single slip system of dislocations was determined by means of $\Gamma$-convergence of the energy expression, see Scardia et al. (2014), Geers et al. (2013). In this work, it was assumed that the dislocations are arranged in wall structures on equally spaced glide planes and that the system is at zero temperature.

Despite all these efforts, no explicit free energy expression has been proposed yet, that is derived from the microscopic properties of the system, and thus includes the anisotropy of the dislocation interaction, the finite system size and the presence of glide planes, and which is valid in different temperature regimes. The aim of this paper is to obtain such a free energy expression. In this contribution, we limit ourselves to straight dislocations with parallel line orientation.

The free energy is derived by systematically coarse-graining the microscopic description of dislocations as used in Discrete Dislocation Dynamics (DDD) simulations. In an earlier paper (Kooiman et al., 2014), we derived the partition function of dislocations for a grand-canonical ensemble of straight and parallel dislocations. In this contribution, we derive the Helmholtz free energy of dislocations from this by means of a Legendre transform. The obtained free energy contains elastic energy and statistical terms, as found earlier by Groma et al. (2006), but yields also a many-body contribution beyond these mean-field terms. It is, to our best knowledge, for the first time that the free energy was derived by coarse-graining only.

The resulting free energy depends on densities of positive and negative dislocations separately for each slip system. This implies that the defect forces in crystal plasticity models (see e.g. Gurtin, 2008) cannot be determined in terms of GND densities alone.

The paper is organized as follows. In Section 2, we discuss the microscopic and macroscopic descriptions of the system. Then, we briefly outline the derivation of the grand-canonical partition function and perform a Legendre transform to obtain the canonical free energy in Eq. (2.22). In Section 3, we discuss the interpretation and limitations of the obtained free energy expression. In Section 4, three special cases are considered in which the free energy expression simplifies considerably, namely a local density approximation (LDA), the zero temperature limit, and equally spaced glide planes. In Section 5 , the connection is made between this work and current dislocation-based crystal plasticity models.

\section{Derivation}

\subsection{Mathematical preliminaries}

In this paper, both two-dimensional and three-dimensional position vectors are used. To avoid confusion, the twodimensional position vector is denoted by $\boldsymbol{s}$ and consists of an $x$ and a $y$-coordinate. Integration over this vector is denoted by $\int d A$. On the other hand, the three-dimensional position vector is denoted by $\boldsymbol{r}$ and consists of an $x, y$ and $z$-coordinate. Integration over the 3D position vector is denoted by $\int d V$.

The line direction $\hat{\xi}$ of the straight and parallel dislocations is parallel to the $\hat{z}$-direction, and the position in this direction is denoted by $z$. Thus, the vector $\boldsymbol{r}$ can be expressed in $\boldsymbol{s}$ and $z$ by $\boldsymbol{r}=\boldsymbol{s}+z \hat{\xi}$, and analogously, the integration over 3D position vectors can be expressed as $\int d V=\int d A \int d z$. See Fig. 1 for a sketch of the coordinate system.

In this work, the cross product on a tensor is interpreted as the cross product on the first index, so the cross product between vector $\boldsymbol{v}$ and second rank tensor $\boldsymbol{A}$ is $(\boldsymbol{v} \times \boldsymbol{A})_{i j}=\epsilon_{i k l} v_{k} A_{l j}$, where $\epsilon$ is the anti-symmetric Levi-Cività tensor. A contraction of a second rank tensor $\boldsymbol{A}$ and a fourth rank tensor $\mathrm{B}$ is defined by $(\boldsymbol{A}: \mathrm{B})_{k l}=A_{i j} B_{i j k l}$, and the trace of a fourth rank tensor $\mathrm{B}$ is $\operatorname{Tr}[\mathrm{B}]=B_{i j i j}$. The symmetric and anti-symmetric parts of a second rank tensor are indicated with a superscript $s$ and a; $\left(\boldsymbol{A}^{\mathrm{s}, \mathrm{a}}\right)_{i j}=\left(A_{i j} \pm A_{j i}\right) / 2$. The $\otimes$-symbol is used to indicate a dyadic product.

In this work, round brackets indicate a function, and square brackets indicate a functional.

Furthermore, Fourier transforms are used multiple times in this contribution. We use the non-unitarian convention here, 


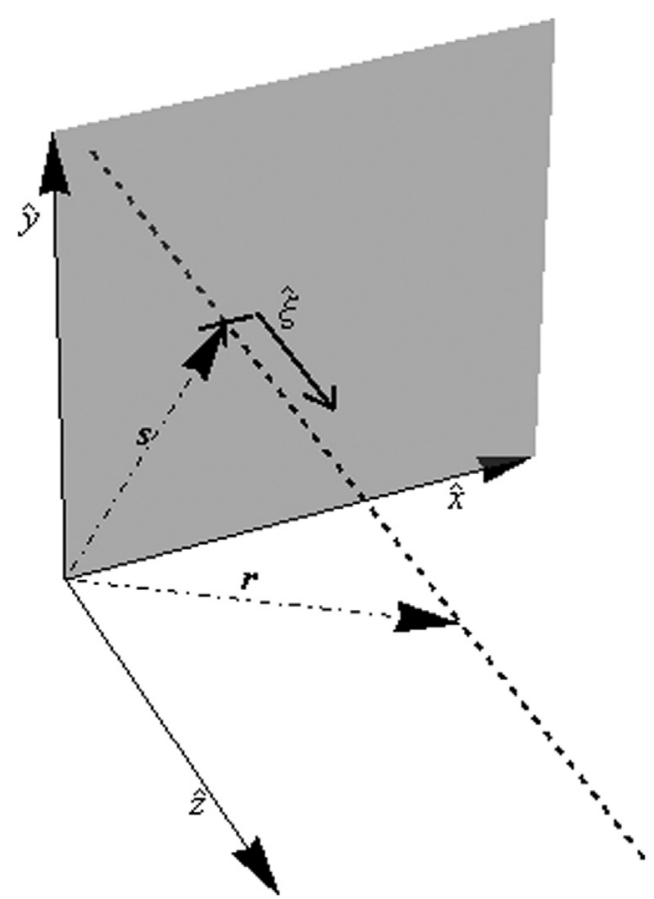

Fig. 1. Sketch of the coordinate system used in this paper.

and hence the 2D Fourier transform is defined by

$$
\mathcal{F}_{2 D}[f(\boldsymbol{s})]\left(\boldsymbol{q}_{2 D}\right)=\tilde{f}\left(\boldsymbol{q}_{2 D}\right)=\int d A f(\boldsymbol{s}) e^{-1 \mathbf{q}_{2 D} \cdot \boldsymbol{s}},
$$

where $\boldsymbol{q}_{2 D}$ is the 2D wave-vector. Consequently, the inverse 2D Fourier transform is defined by

$$
\mathcal{F}_{2 D}^{-1}\left[\tilde{f}\left(\boldsymbol{q}_{2 D}\right)\right](\boldsymbol{s})=\int \frac{d^{2} \boldsymbol{q}_{2 D}}{(2 \pi)^{2}} \tilde{f}\left(\boldsymbol{q}_{2 D}\right) e^{1 \boldsymbol{q}_{2 D} \cdot \boldsymbol{s}} .
$$

The Fourier transform in 3D is defined analogously.

\subsection{Multiscale description of the problem}

Microscale: The microscopic description of our system is closely related to the description of crystals with dislocations used in DDD simulations, see e.g. Van der Giessen and Needleman (1995).

A linear elastic body is considered. The volume of the body is denoted by $\Omega$, and the elastic properties of the matrix material are governed by the fourth order stiffness tensor $\mathbb{C}$ that relates the stress to the strain. For convenience, the fourth order compliance tensor $\mathbb{S}$ is also defined as the inverse of the stiffness tensor: $\mathbb{C}_{i j k l} \mathbb{S}_{k l i, j}=\delta_{i i}, \delta_{j j}$, where the Einstein summation convention is used.

In this linear elastic body, straight and parallel dislocations are embedded. Each dislocation is characterized by its Burgers vector $\boldsymbol{b}$ and the direction of its line vector $\hat{\xi}$. As straight and parallel dislocations are considered, the line vector is equal for all dislocations.

In this study, climb of dislocations is not accounted for. For the static states of the system, this implies that dislocation can only be positioned on discrete glide planes.

The elastic body is furthermore subjected to a boundary deformation $\boldsymbol{u}_{b}$. The dependence of the free energy on the boundary deformation obtained in Kooiman et al. (2014) is implicit. Therefore, two quantities related to $\boldsymbol{u}_{b}$ are defined here. Since the positions of the dislocations are independent of the $z$-coordinate, it only makes sense to consider deformations of the boundary that are independent of $z$ as well.

Hypothetical strain- and stress fields $\varepsilon_{0}$ and $\sigma_{0}$ can be defined as the strain- and stress field one would find in the same elastic body $\Omega$ with the same boundary deformation $\boldsymbol{u}_{b}$, but without dislocations. These fields have to satisfy mechanical equilibrium in the bulk, and it has to match the imposed boundary deformation $\boldsymbol{u}_{b}$ :

$$
\boldsymbol{\varepsilon}_{0} \equiv(\nabla \boldsymbol{u})^{\mathrm{s}}
$$




$$
\begin{aligned}
& \sigma_{0} \equiv \mathbb{C}: \varepsilon_{0} \\
& \nabla \cdot\left(\sigma_{0}\right)=0 \\
& \boldsymbol{u}=\boldsymbol{u}_{b} .
\end{aligned}
$$

Due to Eq. (2.3c), a second field $\Psi_{0}$ can be defined by

$$
\nabla \times \Psi_{0} \equiv \sigma_{0} .
$$

The field $\Psi_{0}$ is thus uniquely defined up to a gradient. The Beltrami stress potential $\Phi_{0}$, as used in Carlson (1966), is related to $\Psi_{0}$ by $\Psi_{0}=\left(\nabla \times \boldsymbol{\Phi}_{0}\right)^{\mathrm{T}}$, where T indicates the transpose. Note that, as $\boldsymbol{u}_{b}$ is independent of the $z$-coordinate, both $\boldsymbol{\varepsilon}_{0}$ and $\boldsymbol{\Psi}_{0}$ are independent of the $z$-coordinate as well.

The field $\Psi_{0}$ turns out to be convenient to work with, as it is related to the Peach-Koehler force on a dislocation line. Namely, a dislocation with Burgers vector $\boldsymbol{b}$ would experience the following Peach-Koehler force in the hypothetical strain field $\varepsilon_{0}$ :

$$
F_{P K, 0}=\int d z\left(\boldsymbol{\sigma}_{0}(\boldsymbol{s}) \cdot \boldsymbol{b}\right) \times \hat{\xi}=\int d z\left(\nabla \times \Psi_{0}(\boldsymbol{s}) \cdot \boldsymbol{b}\right) \times \hat{\xi}=\int d z(\hat{\xi} \cdot \nabla) \Psi_{0}(\boldsymbol{s}) \cdot \boldsymbol{b}-\nabla\left(\hat{\xi} \otimes \boldsymbol{b}: \Psi_{0}(\boldsymbol{s})\right),
$$

which is the integral of the Peach-Koehler force on a dislocation line element, see e.g. Landau and Lifshitz (1975), integrated along the line. The first term vanishes as $\boldsymbol{\Psi}_{0}$ is independent of the $z$-coordinate, and hence $(\hat{\xi} \cdot \nabla) \Psi_{0}(\boldsymbol{s})=\partial_{z} \Psi_{0}(\boldsymbol{s})=0$. Then this expression implies that $\int d z \hat{\xi} \otimes \boldsymbol{b}: \Psi_{0}(\boldsymbol{s}, z)$ can be interpreted as the potential energy of a dislocation with Burgers vector $\boldsymbol{b}$ in the strain field $\boldsymbol{\varepsilon}_{0}$. Therefore, we define the Peach-Koehler potential for dislocations with Burgers vector $\boldsymbol{b}$ by

$$
V_{b, 0}(\boldsymbol{s})=\int d z \hat{\xi} \otimes \boldsymbol{b}: \Psi_{0}(\boldsymbol{s})
$$

This potential is uniquely defined up to a constant. The free energy will only depend on $\boldsymbol{u}_{b}$ via $\boldsymbol{\varepsilon}_{0}$ and $V_{\boldsymbol{b}, 0}$.

Finally, microstates are characterized by the strain field $\varepsilon$ in the body. This strain field has to match the incompatibility imposed by the dislocations and the applied boundary deformation $\boldsymbol{u}_{b}$, but it does not have to be in mechanical equilibrium. This implies that we allow for elastic waves or phonons in the material, on top of a mechanical equilibrium state.

Macroscale: On the macroscopic level, the same elastic body $\Omega$ with the same bare stiffness tensor $\mathbb{C}$ is considered. This body is subjected to the same boundary deformation $\boldsymbol{u}_{b}$. Therefore, in view of Eqs. (2.3) and (2.6), $\boldsymbol{\varepsilon}_{0}$ and $V_{\boldsymbol{b}, 0}$ are also defined on the macroscopic scale. Hence no coarse-graining of the boundary conditions is considered. But rather than discrete dislocation positions, the coarse-grained density profile of dislocations with Burgers vector $\boldsymbol{b}, \rho_{\boldsymbol{b}}(\boldsymbol{s})$, is used as a variable on the macroscopic level.

For the coarse-graining procedure it is more convenient to control the average of the dislocation density profile by controlling the local chemical potential $\mu_{\boldsymbol{b}}(\boldsymbol{s})$ of dislocations with Burgers vector $\boldsymbol{b}$. This is called the grand-canonical ensemble. We refer the reader to Chaikin and Lubensky (1995) for more details. It can be proven that the relation between $\mu_{\boldsymbol{b}}(\boldsymbol{s})$ and $\rho_{\boldsymbol{b}}(\boldsymbol{s})$ is unique, see e.g. Evans (1979). This means that for every density profile one can find the corresponding local chemical potential, and that every local chemical potential corresponds to just one dislocation density profile. Hence once the free energy is known as a functional of the local chemical potential, it can also be obtained in terms of the density profile.

The coarse-graining of dislocation positions can be performed in two ways. First, one can average the density in the glide plane, but keep the discrete character of the glide planes, as depicted in Fig. 2(b). The glide plane positions should then be considered as material parameters. An example of this is worked out in Section 4.2.1, where it is assumed that glide planes are equally spaced, and the spacing $h$ is a material parameter.

Second, the averaging can be done both in the glide plane and in the direction perpendicular to it, as depicted in Fig. 2(c). Then, the glide plane distribution is no longer a material parameter. An example of this averaging is worked out in Section 4.1 .

In both cases, the free energy can be determined by the expression obtained in this paper. In the first case, the density should be zero in between the glide planes, and only take non-zero values at these glide planes. In the second case, there are no restrictions on the density profile.

One could also consider a hybrid version of the above two averaging techniques, where glide planes are smeared out, but not necessarily to a homogeneous profile. This would imply that some regions are almost empty (these are the regions in between the smeared out glide planes), whilst others are more likely to contain a lot of dislocations. Such a hybrid version is not considered here.

\subsection{Coarse-graining method}

In this contribution, the coupling between the microscale and the macroscale is made with averaging techniques from statistical physics. This means that the macroscopic free energy $F$ can be determined from the so-called partition function $Z$, see e.g. Chaikin and Lubensky (1995):

$$
F=-k_{B} T \ln Z \text {. }
$$



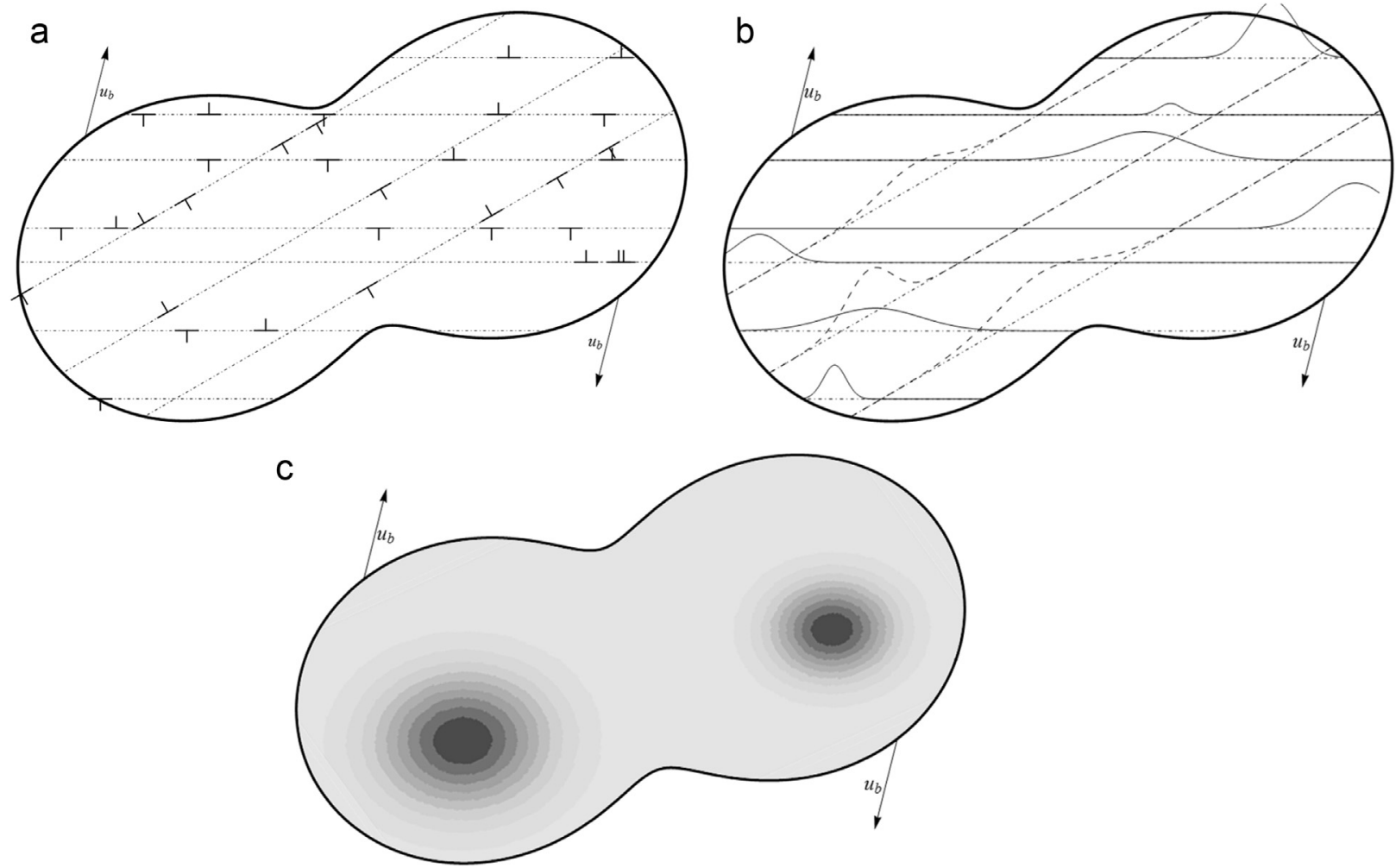

Fig. 2. In Fig. 2(a), the microstate is depicted. The microstate is characterized by the positions of discrete dislocations in an elastic body $\Omega$ subjected to a boundary deformation $\boldsymbol{u}_{b}$. In Fig. 2(b), the macrostate is depicted. The macrostate is characterized by the density of dislocations in the same elastic body $\Omega$, subjected to the same boundary deformation $\boldsymbol{u}_{b}$. The density of dislocations can be defined either on discrete glide planes only, see e.g. Fig. 2(b), or on the whole space, see e.g. Fig. 2(c). The body is held at a fixed temperature $T$.

The partition sum is a sum over all microstates weighted with their Boltzmann weight, and hence it can, in principle, be calculated from the microscopic system description. The Boltzmann weight depends on the macroscopic state variables and is defined as the exponent of minus the energy $E_{\text {micro }}$ of the microstate divided by the thermal energy; $\exp \left(-E_{\text {micro }} / k_{B} T\right)$. Here, $k_{B}$ is the Boltzmann constant, equal to $1.4 \times 10^{-23} \mathrm{~J} / \mathrm{K}$, and $T$ is the absolute temperature. The Boltzmann weight is a measure for how likely a microstate is; microstates with lower energy are more likely, and this preference is stronger at lower temperature $T$. So to conclude, the partition function reads:

$$
Z=\sum_{\text {microstates }} \exp \left(-E_{\text {micro }} / k_{B} T\right)
$$

In an earlier contribution (Kooiman et al., 2014), we already determined an expression for the partition function of a crystal with dislocations. In this contribution, this partition sum is used to obtain an explicit expression for the free energy as a function of the dislocation density.

When the free energy is calculated as in Eq. (2.7), local organization of dislocations is also accounted for. Namely, microstates in which dislocations locally organize themselves in low energy states are more likely, and hence contribute more to the partition function. This lowers the overall free energy in the system.

In this work, average quantities should be interpreted as the statistical average, as opposed to for example a spatial or time averages. Thus, if one would be able to consider multiple microscopic realizations of the same macroscopic system, this is the average one would find. For perfectly ergodic systems, the statistical average matches the time average by definition.

It has been suggested that the ergodicity assumption might not be valid on realistic timescales for dislocation systems. Namely, the behavior in discrete dislocation simulations is very sensitive to the initial dislocation distribution, as the system exhibits high energy barriers. However, it has been shown recently, see Ispánovity et al. (2010), that the statistical average of the behavior in discrete dislocation simulations over many randomly selected initial dislocation distributions gives realistic predictions for the response of large systems.

Therefore, it is assumed here that a statistical average is representative for the macroscopically observed behavior.

\subsection{The partition sum}

To evaluate the partition function in Eq. (2.8), one should sum the Boltzmann weight over all possible microstates. The 
summation over microstates involves an integration over the positions of the dislocations in the glide-plane, and a summation over all possible numbers of dislocations from zero to infinity in each glide plane.

Furthermore, the summation involves an integration over all possible strain fields $\boldsymbol{\varepsilon}$ that match the incompatibility imposed by the dislocations and the applied deformation of the boundary. The integration over fields can be performed by means of a path integral.

The Boltzmann weight follows from the energy of a microstate, see Eq. (2.8). This energy consists of the elastic strain energy and the so-called chemical energy of dislocations. The elastic energy reads

$$
E_{\text {elas }}=\frac{1}{2} \int d V \varepsilon: \mathbb{C}: \varepsilon
$$

This automatically incorporates the energy due to the dislocations and due to boundary conditions, as the elastic strain field in the body matches the incompatibility of the dislocations and the imposed boundary conditions.

The chemical energy of a dislocation with Burgers vector $\boldsymbol{b}$ at position $\boldsymbol{s}$ is minus the chemical potential $\mu_{\boldsymbol{b}}(\boldsymbol{s})$ at that position. The total chemical energy of the dislocations together is thus

$$
E_{\text {chem }}=-\sum_{\boldsymbol{b}}\left(\sum_{k=1}^{N_{\boldsymbol{b}}} \mu_{\boldsymbol{b}}\left(\boldsymbol{s}_{k}\right)\right),
$$

where $N_{\boldsymbol{b}}$ is the number of dislocations with Burgers vector $\boldsymbol{b}$ and $\boldsymbol{s}_{k}$ is the position of the $k$ th dislocation.

The summation over microstates, as described in the previous paragraph, was performed analytically in our earlier work. The only approximation that was made in the derivation is that the system is far from its transition point. In Section 3.3, we will comment further on the implications of this approximation. In this section, the results for the partition function and dislocation density of Kooiman et al. (2014) are summarized.

The partition function is most conveniently expressed in terms of the average dislocation density, although it is a functional of the chemical potential, and not of the dislocation density. The partition function and dislocation density obtained in Kooiman et al. (2014) read

$$
\begin{aligned}
& Z\left[\mu_{\boldsymbol{b}}(\boldsymbol{s}), \boldsymbol{u}_{b}, T\right]= \exp \left[\frac{1}{2 k_{B} T} \int d V \boldsymbol{\varepsilon}_{0}: \mathbb{C}: \boldsymbol{\varepsilon}_{0}+\sum_{\boldsymbol{b}} \int d A \rho_{\boldsymbol{b}}(\boldsymbol{s})+\frac{1}{2 k_{B} T} \sum_{\boldsymbol{b}, \boldsymbol{b}^{\prime}} \int d \operatorname{AdA}^{\prime} \rho_{\boldsymbol{b}}(\boldsymbol{s}) u_{\boldsymbol{b}, \boldsymbol{b},}\left(\boldsymbol{s}, \boldsymbol{s}^{\prime}\right) \rho_{\boldsymbol{b},}\left(\boldsymbol{s}^{\prime}\right)\right. \\
&+\sum_{\boldsymbol{b}} \frac{1}{2 k_{B} T} \int d A \rho_{\boldsymbol{b}}(\boldsymbol{s}) u_{\mathrm{eff}, \boldsymbol{b}, \boldsymbol{b}}(\mathbf{0}) \\
&\left.-\frac{1}{2} \operatorname{Tr}\left[\ln \left[\mathrm{I} \delta\left(\boldsymbol{s}-\boldsymbol{s}^{\prime}\right)+\frac{1}{k_{B} T} \sum_{\boldsymbol{b}} \int d z d z^{\prime} \mathrm{G}_{0}\left(\boldsymbol{s}, z ; \boldsymbol{s}^{\prime}, z^{\prime}\right): \rho_{\boldsymbol{b}}\left(\boldsymbol{s}^{\prime}\right) \hat{\xi} \otimes \boldsymbol{b} \otimes \hat{\xi} \otimes \boldsymbol{b}\right]\right]\right] \\
& \rho_{\boldsymbol{b}}(\boldsymbol{s})=\rho_{\boldsymbol{b}, 0}(\boldsymbol{s}) \exp \left[\frac{\mu_{\boldsymbol{b}}(\boldsymbol{s})-V_{\boldsymbol{b}, 0}(\boldsymbol{s})}{k_{B} T}-\frac{1}{k_{B} T} \sum_{\boldsymbol{b}^{\prime}} \int d A u_{\boldsymbol{b}, \boldsymbol{b},}\left(\boldsymbol{s}, \boldsymbol{s}^{\prime}\right) \rho_{\boldsymbol{b}^{\prime}}\left(\boldsymbol{s}^{\prime}\right)-\frac{1}{2 k_{B} T} u_{\mathrm{eff}, \boldsymbol{b}, \boldsymbol{b}}(\mathbf{0})\right]
\end{aligned}
$$

where $\rho_{\boldsymbol{b}, 0}(\boldsymbol{s})$ is a parameter indicating glide planes when these are defined on the macroscopic scale. In this case, $\rho_{\boldsymbol{b}, 0}(\boldsymbol{s})$ is zero in those points where there is no discrete glide plane for dislocations with Burgers vector $\boldsymbol{b}$ and a Dirac delta function at
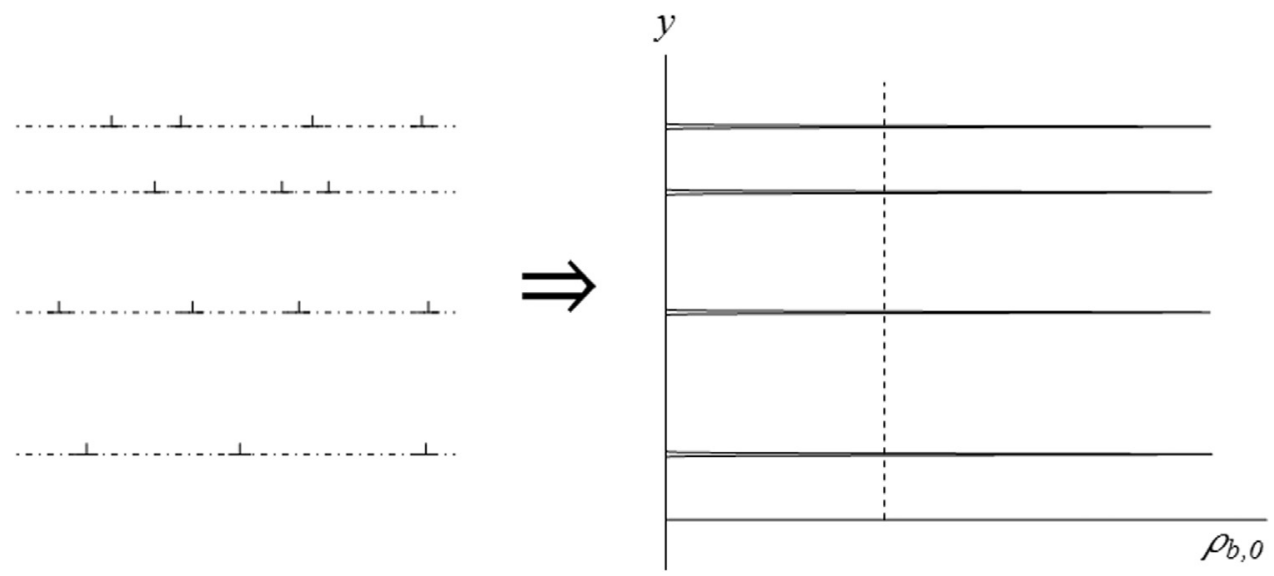

Fig. 3. When glide planes are defined on the macroscopic scale (as in Fig. 2(b)), the parameter $\rho_{\boldsymbol{b}, 0}(\boldsymbol{s})$ indicates the presence of glide planes (solid line). When glide planes are not defined (as in Fig. 2(c)), $\rho_{\boldsymbol{b}, 0}(\boldsymbol{s})$ is a spatial constant (dashed line). 
the glide planes, see Fig. 3. When no discrete glide planes are considered, as in Fig. 2(c), the parameter $\rho_{\boldsymbol{b}, 0}(\boldsymbol{s})$ is a spatial constant. The trace runs over both discrete and continuous indices. The natural logarithm in the last term is the logarithm of an operator, and not just the logarithm of the components. This means that the last term in Eq. (2.11a) can be written as the sum of the logarithm of all eigenvalues of the operator $I \delta\left(\boldsymbol{s}-\boldsymbol{s}^{\prime}\right)+\left(1 / k_{B} T\right) \sum_{\boldsymbol{b}} \int d z d z^{\prime} G_{0}\left(\boldsymbol{s}, z ; \boldsymbol{s}^{\prime}, z^{\prime}\right): \rho_{\boldsymbol{b}}\left(\boldsymbol{s}^{\prime}\right) \hat{\xi} \otimes \boldsymbol{b} \otimes \hat{\xi} \otimes \boldsymbol{b}$.

The dependence on the boundary deformation $\boldsymbol{u}_{b}$ is via $V_{\boldsymbol{b}, 0}$, and the functions $G_{0}\left(\boldsymbol{r} ; \boldsymbol{r}^{\prime}\right), u_{\boldsymbol{b}, \boldsymbol{b}},\left(\boldsymbol{s}, \boldsymbol{s}^{\prime}\right)$ and $u_{\mathrm{eff}, \boldsymbol{b}, \boldsymbol{b}},\left(\boldsymbol{s}, \boldsymbol{s}^{\prime}\right)$ are the bare Greens function and the bare and effective interaction between dislocations, that will be defined next.

The function $u_{\boldsymbol{b}, \boldsymbol{b}},\left(\boldsymbol{s}, \boldsymbol{s}^{\prime}\right)$ is the interaction energy of two dislocations with Burgers vector $\boldsymbol{b}$ and $\boldsymbol{b}^{\prime}$ at positions $\boldsymbol{s}$ and $\boldsymbol{s}^{\prime}$ in finite space. The interaction energy is the extra energy that is needed to create a dislocation in the material while another dislocation is already present. The interaction energy can be written in terms of the Green's function $G_{0, i j k l}\left(\boldsymbol{r}, \boldsymbol{r}^{\prime}\right)$ by

$$
u_{\boldsymbol{b}, \boldsymbol{b}}\left(\boldsymbol{s}, \boldsymbol{s}^{\prime}\right)=\int d z d z^{\prime} \hat{\xi} \otimes \boldsymbol{b}: \mathrm{G}_{0, i j i, j}\left(\boldsymbol{s}, z ; \boldsymbol{s}^{\prime}, z^{\prime}\right): \hat{\xi} \otimes \boldsymbol{b}^{\prime}
$$

In Kooiman et al. (2014), it was found that the fourth order tensor $G_{0}\left(\boldsymbol{r}, \boldsymbol{r}^{\prime}\right)$ is such that, for all fields $\Psi(\boldsymbol{r})$ that are divergence free $(\nabla \cdot \Psi=0)$ and for which $\nabla \times \Psi(\boldsymbol{r})$ is symmetric, one finds

$$
\int d V \nabla \times \boldsymbol{\Psi}(\boldsymbol{r}): \mathbb{S}: \nabla \times \Psi(\boldsymbol{r})=\int d V d V^{\prime} \Psi(\boldsymbol{r}): \mathrm{G}_{0}^{-1}\left(\boldsymbol{r}, \boldsymbol{r}^{\prime}\right): \Psi\left(\boldsymbol{r}^{\prime}\right) .
$$

Furthermore, $\mathrm{G}_{0}^{-1}\left(\boldsymbol{r}, \boldsymbol{r}^{\prime}\right)$ is defined such that the RHS of this equation is infinite for all fields that are either not divergence free or for which $\nabla \times \Psi(\boldsymbol{r})$ is not symmetric. The defining equation (2.13) of $\mathrm{G}_{0}^{-1}\left(\boldsymbol{r}, \boldsymbol{r}^{\prime}\right)$ is thus rather implicit and cannot be written in an easier form. Namely, the spatial integration acts over a finite volume, and hence the above cannot be written in a partial differential equation for $G_{0}\left(\boldsymbol{r}, \boldsymbol{r}^{\prime}\right)$. However, in this form, Eq. (2.13) incorporates the effect of finite space and can be used to derive a differential equation for the dislocation density profile.

It was shown that in infinite space and for an isotropic material, Eqs. (2.12) and (2.13) yield the interaction energy per unit length between dislocations as known from the literature, see e.g. Hirth and Lothe (1982) and Raabe et al. (2004):

$$
\begin{aligned}
& \tilde{\mathrm{G}}_{0, i j k l}(\boldsymbol{q})=\frac{\mu}{q^{2}}\left(Q_{i k} Q_{j l}+R_{i l} R_{j k}+\frac{2 \nu}{1-\nu} R_{i j} R_{k l}\right), \\
& \Rightarrow \frac{u_{\boldsymbol{b}, \boldsymbol{b}}(\Delta \boldsymbol{s})}{L}=\frac{\mu}{2 \pi}\left(\left(\boldsymbol{b} \cdot \hat{\boldsymbol{\xi}} \boldsymbol{b}^{\prime} \cdot \hat{\boldsymbol{\xi}}+\frac{(\boldsymbol{b} \times \hat{\boldsymbol{\xi}}) \cdot\left(\boldsymbol{b}^{\prime} \times \hat{\xi}\right)}{1-\nu}\right) \ln \left(\frac{|\Delta \boldsymbol{s}|}{s_{0}}\right)+\frac{(\boldsymbol{b} \times \hat{\boldsymbol{\xi}}) \cdot \Delta \boldsymbol{s}\left(\boldsymbol{b}^{\prime} \times \hat{\boldsymbol{\xi}}\right) \cdot \Delta \boldsymbol{s}}{(1-\nu)|\Delta \boldsymbol{s}|^{2}}\right)
\end{aligned}
$$

where $Q_{i j}=\delta_{i j}-q_{i} q_{j} / q^{2}$ and $R_{i j}=\epsilon_{i j k} q_{k} / q$, where $\epsilon_{i j k}$ is the anti-symmetric Levi-Cività tensor. Furthermore, $\mu$ is the shear modulus, and $\nu$ is the Poisson's ratio, such that the isotropic compliance tensor reads $\mathbb{S}_{i j i, j},=2 \mu\left(\delta_{i i}, \delta_{j j},-\nu /(1+\nu) \delta_{i j} \delta_{i, j}\right)$.

The function $u_{\text {eff, }, \boldsymbol{b}, \boldsymbol{b}},\left(\boldsymbol{s}, \boldsymbol{s}^{\prime}\right)$ in Eq. (2.11b) can be interpreted as the effective interaction energy between two dislocations, in which the effect of screening is incorporated as well. It can analogously be written as

$$
u_{\mathrm{eff}, \boldsymbol{b}, \boldsymbol{b},}\left(\boldsymbol{s}, \boldsymbol{s}^{\prime}\right)=\int d z d z^{\prime} \hat{\boldsymbol{\xi}} \otimes \boldsymbol{b}: \mathrm{G}_{i j i, j}\left(\boldsymbol{s}, z ; \boldsymbol{s}^{\prime}, z^{\prime}\right): \hat{\boldsymbol{\xi}} \otimes \boldsymbol{b}^{\prime},
$$

where it was shown in previous work that the two tensors $G_{0}$ and $G$ are related by the implicit relation

$$
\mathrm{G}\left(\boldsymbol{r}, \boldsymbol{r}^{\prime}\right)=\mathrm{G}_{0}\left(\boldsymbol{r}, \boldsymbol{r}^{\prime}\right)+\sum_{\boldsymbol{b}} \int d z d z^{\prime} \int d A^{\prime \prime} \mathrm{G}\left(\boldsymbol{r} ; \boldsymbol{s}^{\prime \prime}, z\right): \rho_{\boldsymbol{b}}\left(\boldsymbol{s}^{\prime \prime}\right) \hat{\xi} \otimes \boldsymbol{b} \otimes \hat{\xi} \otimes \boldsymbol{b}: \mathrm{G}_{0}\left(\boldsymbol{s}^{\prime \prime}, z^{\prime} ; \boldsymbol{r}^{\prime}\right) .
$$

Then, $u_{\mathrm{eff}, \boldsymbol{b}, \boldsymbol{b}}(\mathbf{0}) / 2$ can be interpreted as the effective self-energy of a dislocation with Burgers vector $\boldsymbol{b}$. Note that the relation between $\rho_{\boldsymbol{b}}(\boldsymbol{s})$ and $\mu_{\boldsymbol{b}}(\boldsymbol{s})$ in Eq. (2.11b) is an implicit expression as the terms in the exponent depend on the density. The dependence of $Z$ on the local chemical potential $\mu_{\boldsymbol{b}}(\boldsymbol{s})$ in Eq. (2.11a) is therefore implicit as well.

The average density profile for a given chemical potential can be determined directly from Eq. (2.11b) without using a free energy expression in terms of the density profile. However, the free energy does not only yield the equilibrium dislocation configuration, but also other static properties of the system, and the driving forces for dislocation dynamics.

From Eq. (2.7) and (2.11a), the thermodynamic potential can be determined:

$$
\begin{aligned}
\Omega\left[\mu_{\boldsymbol{b}}(\boldsymbol{s}), \boldsymbol{u}_{b}, T\right] \equiv & -k_{B} T \ln \left(Z\left[\mu_{\boldsymbol{b}}(\boldsymbol{s}), \boldsymbol{u}_{b}, T\right]\right)=\frac{1}{2} \int d V \boldsymbol{\varepsilon}_{0}: \mathbb{C}: \boldsymbol{\varepsilon}_{0} \\
& -\frac{1}{2} \sum_{\boldsymbol{b}, \boldsymbol{b}^{\prime}} \int d A d A^{\prime} \rho_{\boldsymbol{b}}(\boldsymbol{s}) u_{\boldsymbol{b}, \boldsymbol{b}}\left(\boldsymbol{s}, \boldsymbol{s}^{\prime}\right) \rho_{\boldsymbol{b}}\left(\boldsymbol{s}^{\prime}\right)-k_{B} T \sum_{\boldsymbol{b}} \int d A \rho_{\boldsymbol{b}}(\boldsymbol{s})-\frac{1}{2} \sum_{\boldsymbol{b}} \int d A \rho_{\boldsymbol{b}}(\boldsymbol{s}) u_{\mathrm{eff}, \boldsymbol{b}, \boldsymbol{b}}(\mathbf{0}) \\
& +\frac{k_{B} T}{2} \operatorname{Tr}\left[\ln \left(\operatorname{I} \delta\left(\boldsymbol{s}-\boldsymbol{s}^{\prime}\right)+\frac{1}{k_{B} T} \sum_{\boldsymbol{b}} \int d z d z^{\prime} \mathrm{G}_{0}\left(\boldsymbol{s}, z ; \boldsymbol{s}^{\prime}, z^{\prime}\right): \rho_{\boldsymbol{b}}\left(\boldsymbol{s}^{\prime}\right) \hat{\boldsymbol{\xi}} \otimes \boldsymbol{b} \otimes \hat{\boldsymbol{\xi}} \otimes \boldsymbol{b}\right)\right] .
\end{aligned}
$$




\subsection{Legendre transform}

To obtain the free energy from the thermodynamic potential of the grand-canonical ensemble, the following Legendre transform is applied

$$
F\left[\rho_{\boldsymbol{b}}(\boldsymbol{s}), \boldsymbol{u}_{b}, T\right]=\Omega\left[\mu_{\boldsymbol{b}}\left[\rho_{\boldsymbol{b}}(\boldsymbol{s}), \boldsymbol{u}_{b}, T ; \boldsymbol{s}\right], \boldsymbol{u}_{b}, T\right]+\sum_{\boldsymbol{b}} \int d A \rho_{\boldsymbol{b}}(\boldsymbol{s}) \mu_{\boldsymbol{b}}\left[\rho_{\boldsymbol{b}}(\boldsymbol{s}), \boldsymbol{u}_{b}, T ; \boldsymbol{s}\right],
$$

where $\delta \Omega / \delta \mu_{\boldsymbol{b}}(\boldsymbol{s})=\rho_{\boldsymbol{b}}(\boldsymbol{s})$. It can be checked that this is indeed the case by taking the derivative with respect to $\mu_{\boldsymbol{b}}(\boldsymbol{s})$ in Eq. (2.17). To perform this transformation, it is thus necessary to find the chemical potential in terms of the dislocation density profile. This can be done by inverting Eq. (2.11b):

$$
\mu_{\boldsymbol{b}}(\boldsymbol{s})=k_{B} T \ln \left(\frac{\rho_{\boldsymbol{b}}(\boldsymbol{s})}{\rho_{0, \boldsymbol{b}}(\boldsymbol{s})}\right)+V_{\boldsymbol{b}, 0}(\mathbf{s})+\sum_{\boldsymbol{b}^{\prime}} \int d A^{\prime} u_{\boldsymbol{b}, \boldsymbol{b}},\left(\boldsymbol{s}, \boldsymbol{s}^{\prime}\right) \rho_{\boldsymbol{b}},\left(\mathbf{s}^{\prime}\right)+\frac{1}{2} u_{\mathrm{eff}, \boldsymbol{b}, \boldsymbol{b}}(\mathbf{0})
$$

Combining Eqs. (2.17), (2.18) and (2.19) yields:

$$
\begin{aligned}
F\left[\rho_{\boldsymbol{b}}(\boldsymbol{s}), \boldsymbol{u}_{b}, T\right]= & \frac{1}{2} \int d V \boldsymbol{\varepsilon}_{0}: \mathbb{C}: \boldsymbol{\varepsilon}_{0}+\frac{1}{2} \sum_{\boldsymbol{b}, \boldsymbol{b}^{\prime}} \int d A d A^{\prime} \rho_{\boldsymbol{b}}(\boldsymbol{s}) u_{\boldsymbol{b}, \boldsymbol{b}}\left(\boldsymbol{s}, \boldsymbol{s}^{\prime}\right) \rho_{\boldsymbol{b}}\left(\boldsymbol{s}^{\prime}\right) \\
& +\sum_{\boldsymbol{b}} \int d A \rho_{\boldsymbol{b}}(\boldsymbol{s}) V_{\boldsymbol{b}, 0}(\boldsymbol{s})+k_{B} T \sum_{\boldsymbol{b}} \int d A \rho_{\boldsymbol{b}}(\boldsymbol{s})\left(\log \left(\frac{\rho_{\boldsymbol{b}}(\boldsymbol{s})}{\rho_{\boldsymbol{b}, 0}}\right)-1\right) \\
& +\frac{k_{B} T}{2} \operatorname{Tr}\left[\ln \left(\mathrm{I} \delta\left(\boldsymbol{s}-\boldsymbol{s}^{\prime}\right)+\frac{1}{k_{B} T} \sum_{\boldsymbol{b}} \int d z d z^{\prime} \mathrm{G}_{0}\left(\boldsymbol{s}, z ; \boldsymbol{s}^{\prime}, z^{\prime}\right): \rho_{\boldsymbol{b}}\left(\boldsymbol{s}^{\prime}\right) \hat{\xi} \otimes \boldsymbol{b} \otimes \hat{\xi} \otimes \boldsymbol{b}\right)\right] .
\end{aligned}
$$

The first three terms can be recombined in a single term that captures the mean field contributions of the system. This term can be written in terms of the mean field elastic strain, $\boldsymbol{\varepsilon}_{\mathrm{mf}}$, defined by

$$
\begin{aligned}
& \nabla \times \boldsymbol{\beta}^{p}=-\sum_{\boldsymbol{b}} \hat{\boldsymbol{\xi}} \boldsymbol{b} \rho_{\boldsymbol{b}}(\boldsymbol{s}) \\
& \boldsymbol{\varepsilon}_{\mathrm{mf}}=\left(\nabla \boldsymbol{u}-\boldsymbol{\beta}^{p}\right)^{s} \\
& \left.\begin{array}{l}
\boldsymbol{\sigma}_{\mathrm{mf}}=\mathbb{C}: \boldsymbol{\varepsilon}_{\mathrm{mf}} \\
\nabla \cdot \boldsymbol{\sigma}_{\mathrm{mf}}=0
\end{array}\right\} \text { in the bulk } \\
& \left.\begin{array}{l}
\boldsymbol{u}=\boldsymbol{u}_{b} \\
\boldsymbol{\beta}^{p}=0
\end{array}\right\} \quad \text { on the boundary. }
\end{aligned}
$$

Note that $\boldsymbol{\varepsilon}_{\mathrm{mf}}(\boldsymbol{r})$ is a strongly non-local functional of the dislocation density profiles $\rho_{\boldsymbol{b}}(\boldsymbol{s})$, as a dislocation at position $\boldsymbol{s}$ causes a strain field in the whole volume, and not only at $\boldsymbol{s}$. The free energy in terms of $\boldsymbol{\varepsilon}_{\mathrm{mf}}$ reads (see Appendix A for the derivation):

$$
\begin{aligned}
F\left[\rho_{\boldsymbol{b}}(\boldsymbol{s}), \boldsymbol{u}_{b}, T\right]= & \frac{1}{2} \int d V \boldsymbol{\varepsilon}_{\mathrm{mf}}: \mathbb{C}: \boldsymbol{\varepsilon}_{\mathrm{mf}}+k_{B} T \sum_{\boldsymbol{b}} \int d A \rho_{\boldsymbol{b}}(\boldsymbol{s})\left(\log \left(\frac{\rho_{\boldsymbol{b}}(\boldsymbol{s})}{\rho_{\boldsymbol{b}, 0}}\right)-1\right) \\
& +\frac{k_{B} T}{2} \operatorname{Tr}\left[\ln \left(\mathrm{I} \delta\left(\boldsymbol{s}-\boldsymbol{s}^{\prime}\right)+\frac{1}{k_{B} T} \sum_{\boldsymbol{b}} \int d z d z^{\prime} \mathrm{G}_{0}\left(\boldsymbol{s}, z ; \boldsymbol{s}^{\prime}, z^{\prime}\right): \rho_{\boldsymbol{b}}\left(\boldsymbol{s}^{\prime}\right) \hat{\xi} \otimes \boldsymbol{b} \otimes \hat{\xi} \otimes \boldsymbol{b}\right)\right] .
\end{aligned}
$$

The dependence of the free energy on the boundary deformation $\boldsymbol{u}_{b}$ in this expression enters via the boundary conditions on $\boldsymbol{\varepsilon}_{\mathrm{mf}}$ in Eqs. (2.21). The dependence on the finite volume is via $\boldsymbol{\varepsilon}_{\mathrm{mf}}$ and $\mathrm{G}_{0}\left(\boldsymbol{r} ; \boldsymbol{r}^{\prime}\right)$, as the finite volume appears explicitly in the definitions in Eqs. (2.21) and (2.13) respectively. Analogously, the free energy expression in Eq. (2.20) depends on the loading via $V_{\boldsymbol{b}, 0}(\boldsymbol{s})$, and on the finite volume via both $V_{\boldsymbol{b}, 0}(\boldsymbol{s})$ and $\mathrm{G}_{0}\left(\boldsymbol{r} ; \boldsymbol{r}^{\prime}\right)$.

The expressions in Eqs. (2.20) and (2.13) are equivalent. The advantage of the former is that it is an explicit functional of the dislocation density profiles $\rho_{\boldsymbol{b}}(\boldsymbol{s})$. Namely, the interaction potential $u_{\boldsymbol{b}, \boldsymbol{b}},\left(\boldsymbol{s}, \boldsymbol{s}^{\prime}\right)$ and the Green's function $\mathrm{G}_{0}(\boldsymbol{r}, \boldsymbol{r})$, as implicitly defined in Eqs. (2.12) and (2.13), are independent of the dislocation density profile. The advantage of the latter is that the first contribution in terms of the mean-field elastic strain, is quite common in literature. For example, this term is equivalent to the contribution to the internal power in the work of Gurtin, see Eq. (3.1) in Gurtin (2008) that relates stress to the rate of elastic distortion. Therefore, the latter will be used primarily for comparison with literature. The rest of the paper is devoted to the interpretation of the free energy expressions in Eqs. (2.20) and (2.22), and examples of how they can be used. 


\section{Interpretation of the free energy expression}

\subsection{Interpretation of different contributions}

In this section, the different terms of the obtained expression for the free energy, Eq. (2.22), are interpreted.

The first term in Eq. (2.22) can be interpreted as the mean field elastic energy:

$$
F_{\text {elas }} \equiv \frac{1}{2} \int d V \varepsilon_{\mathrm{mf}}: \mathbb{C}: \boldsymbol{\varepsilon}_{\mathrm{mf}} \text {. }
$$

The strain field $\boldsymbol{\varepsilon}_{\mathrm{mf}}$ is the strain field in the body due to boundary loading and the average dislocation density. Consequently, $F_{\text {elas }}$ contains three effects: first, the elastic energy, due to loading, which is there when no dislocations are present, second, the interaction between net amounts of dislocations due to the strain field that they produce, and third the influence of mechanical loading on dislocations. These three effects are separated in Eq. (2.20), and one can define the background elastic energy, the two-body interaction energy and the loading energy by

$$
\begin{aligned}
& F_{\text {background }} \equiv \frac{1}{2} \int d V \varepsilon_{0}: \mathbb{C}: \boldsymbol{\varepsilon}_{0} \\
& F_{2 b} \equiv \frac{1}{2} \sum_{\boldsymbol{b}, \boldsymbol{b}^{\prime}} \int d A d A^{\prime} \rho_{\boldsymbol{b}}(\boldsymbol{s}) u_{\boldsymbol{b}, \boldsymbol{b}}\left(\boldsymbol{s}, \boldsymbol{s}^{\prime}\right) \rho_{\boldsymbol{b}}\left(\boldsymbol{s}^{\prime}\right) \\
& F_{\text {loading }} \equiv \sum_{\boldsymbol{b}} \int d A \rho_{\boldsymbol{b}}(\boldsymbol{s}) V_{\boldsymbol{b}, 0}(\boldsymbol{s}) .
\end{aligned}
$$

The definition of the two-body contribution $F_{2 b}$ is motivated by $u_{\boldsymbol{b}, \boldsymbol{b}},\left(\boldsymbol{s}, \boldsymbol{s}^{\prime}\right)$, which is the interaction energy of two discrete dislocations with Burgers vector $\boldsymbol{b}$ and $\boldsymbol{b}^{\prime}$ at positions $\boldsymbol{s}$ and $\boldsymbol{s}^{\prime}$, respectively. This expression for the two-body interaction is a mean-field expression, as the two-body density is written as the product of one-body densities, see e.g. Groma (1997). The mean field contribution is expected to be the leading order term for the interaction between dislocations.

Furthermore, the definition of the loading contribution $F_{\text {loading }}$ is motivated by Eq. (2.6), which shows that $V_{\boldsymbol{b}, 0}(\boldsymbol{s})$ is the potential energy corresponding to the Peach-Koehler force that a dislocation would feel in an otherwise dislocation-free body where $\boldsymbol{u}_{b}$ is the imposed boundary deformation. Hence $V_{\boldsymbol{b}, 0}(\boldsymbol{s})$ is the work that the external Peach-Koehler force has performed to move a dislocation with Burgers vector $\boldsymbol{b}$ from infinity to position $\boldsymbol{s}$.

The second term in Eq. (2.22) is a statistical contribution of the dislocations. This is what the free energy of the dislocations would be if they would not interact (i.e. if it would be a sort of an ideal gas). Following the work of Evans for inhomogeneous systems (Evans, 1979), this contribution reads

$$
F_{\text {stat }} \equiv k_{B} T \sum_{\boldsymbol{b}} \int d A \rho_{\boldsymbol{b}}(\boldsymbol{s})\left(\log \left(\frac{\rho_{\boldsymbol{b}}(\boldsymbol{s})}{\rho_{\boldsymbol{b}, 0}}\right)-1\right) .
$$

A similar contribution is also accounted for by Groma et al. (2006), based on phenomenological arguments.

The third term in Eq. (2.22) is a truly many-body contribution that arises from the coarse description of the system:

$$
F_{m b} \equiv \frac{k_{B} T}{2} \operatorname{Tr}\left[\ln \left(\operatorname{I} \delta\left(\boldsymbol{s}-\boldsymbol{s}^{\prime}\right)+\frac{1}{k_{B} T} \sum_{\boldsymbol{b}} \int d z d z^{\prime} \mathrm{G}_{0}\left(\boldsymbol{s}, z ; \boldsymbol{s}^{\prime}, z^{\prime}\right): \rho_{\boldsymbol{b}}\left(\boldsymbol{s}^{\prime}\right) \hat{\xi} \otimes \boldsymbol{b} \otimes \hat{\boldsymbol{\xi}} \otimes \boldsymbol{b}\right)\right]
$$

This term accounts for the effect of the local arrangement of dislocations. As we found in our earlier work (Kooiman et al., 2014), Statistically Stored Dislocations are likely to arrange themselves in pairs, which are low energy structures. This was confirmed by DDD simulations in Groma et al. (2010). The local arrangement of dislocations can reduce the energy of the system, and thus yields a correction to the two-body interaction term that accounts only for the leading order mean field term. This correction is what is accounted for in the many-body contribution.

\subsection{Influence of dislocation length}

In the derivation in Kooiman et al. (2014), and hence in the above derivation, it was used that the dislocations are straight and of infinite length. The question thus arises to what extent the results obtained in this paper are also applicable to dislocations that are curved.

To this end, we consider a slab of the material with a thickness $L$ in which dislocations can be considered approximately straight. To be more precise, we consider the case where the radius of curvature of the dislocation line is substantially larger than the slab thickness $L$.

The dependence on the slab thickness $L$ of the free energy expression in Eq. (2.22) is non-trivial, as both volume and surface integrals are present. These differ by a factor $L$. The origin of this different dependency is that on the microscale, the 
elastic energy scales linearly with the dislocation length, see Eq. (2.9), while the chemical energy in Eq. (2.10) and the number of possible arrangements are independent of the dislocation length, as the arrangement of straight and parallel dislocations is described points in a two-dimensional plane (independent of the $z$-coordinate).

There is no experimental value for the typical radius of curvature of dislocations, as measurements on individual dislocations are notoriously difficult. Even so, one can give a rough estimate on the absolute lower bound for this radius. Namely, this radius could not be shorter than the lattice spacing, and moreover, it would not make sense to model dislocations with a radius of curvature shorter than a few Burger's vectors as straight and infinitely long. Therefore, one can safely assume that the slab thickness $L$ is longer than one Burger's vector: $|\boldsymbol{b}|<L$.

To study the influence of $L$ on the qualitative behavior of the system, it is most convenient to study the free energy expression in Eq. (2.22) in units of $k_{B} T$. When no external loading is applied, the elastic contribution $F_{\text {elas }}$ reduces to the twobody contribution $F_{2 b}$, see Eqs. (3.2), which is proportional to $\Xi \equiv \mu b^{2} L / k_{B} T$, cf. Eq. (2.14b). It can be shown that also in the many body contribution, the term in the logarithm depends on the coupling parameter $\Xi$. The overall prefactor of both the statistical and many body contribution is 1 . Physical results thus only depend on the value of the coupling parameter $\Xi$.

The parameter $\Xi$ is a dimensionless coupling parameter that compares the typical interaction energy with the thermal energy. When $\Xi$ is much larger than 1 , the energetic effects are more important than thermal fluctuations. As for aluminium at room temperature, the shear modulus, the length of the Burgers vector and $k_{B} T$ are known. ${ }^{1}$ Therefore, the lower bound of $\Xi$ is $\Xi>1.6 \times 10^{2}$ (from the lower bound of $L$ estimated above). This implies that, in aluminum at room temperature, the characteristic energy of the dislocation interaction $\left(\mu b^{2} L\right)$ dominates over the characteristic energy of the thermal lattice vibrations $\left(k_{B} T\right)$, even at the lower bound for $L$. It is expected that this holds for other metals as well. Therefore, the exact value of the slab thickness $L$ will not affect qualitatively the behavior of dislocations.

In the limit of infinitely long dislocations, $\Xi$ goes to infinity. As $\Xi$ is already large for dislocations in a reasonably thick slab, it can be concluded that they will behave as if they are infinitely long.

\subsection{Limitations}

The grand canonical partition function in Eq. (2.11a) was derived using a Gaussian approximation for the partition function. This approximation is accurate far away from transition points, around which the macroscopic behavior of the material changes qualitatively. An example of a transition point in this model is dislocation-mediated melting, see for example the work of Kosterlitz and Thouless (1973) and Mizushima (1960). Below a certain critical temperature, dislocations tend to arrange themselves in tightly bound pairs and the material is considered to be solid. Above this temperature, dislocations are able to move more or less freely through the crystal.

Another example is of a transition point is the change from elastic to plastic material response.

The proposed free energy expression is, because of this Gaussian approximation, inaccurate close to transition points of dislocation systems. To study the properties of the system close to the transition, it is better to use the more involved partition function without Gaussian approximation obtained in Kooiman et al. (2014). For example, renormalization group theory can be used to study transition points, even for complicated partition functions. Another option is to use more sophisticated approximation techniques instead of a single Gaussian approximation. For example, the Villain approximation (Kleinert, 1989) is accurate in a much larger range of parameters, but this approximation is computationally more involved.

\section{Application to specific cases}

In this section, the free energy expression in Eq. (2.22) will be applied to three specific cases. First, a Local Density Approximation (LDA) will be considered, as this is often assumed in literature. The validity of the LDA is examined and the resulting energy expression is compared to literature. Second, the zero temperature limit of the free energy will be studied. And third, a completely regular, equally spaced arrangement of glide planes will be considered in the zero temperature limit.

\subsection{Local density approximation (LDA)}

In several dynamical models, a local form is assumed for the free energy. This means that the free energy is the spatial integral of a free energy density, where the latter is only a function of the dislocation density at that point, rather than a functional of the full dislocation density profile. Such an expression is easier to work with. Furthermore, the exact position of glide planes is not used as a material parameter in these models, and moreover, these are almost impossible to obtain experimentally.

In this section, local approximations and gradient corrections for the two body- and the many body contribution are determined from standard expressions from density functional theory, introduced in Eqs. (4.1) and (4.2). The glide planes are smeared out completely, as depicted in Fig. 2(c). For simplicity, only a single slip system is considered. Furthermore, it is assumed that the infinite space solution can be used for the interaction potential $u_{\boldsymbol{b}, \boldsymbol{b}},\left(\boldsymbol{s}-\boldsymbol{s}^{\prime}\right)$ and for the Green's function

\footnotetext{
${ }^{1}$ For aluminium at room temperature, $\mu=26 \mathrm{GPa}, \nu=.33, b=2.9 \AA$ (see Smallman and Ngan, 2011) and $k_{B} T=4 \times 10^{-21} \mathrm{~J}$ at $298 \mathrm{~K}$.
} 
$\mathrm{G}_{0}\left(\boldsymbol{r}-\boldsymbol{r}^{\prime}\right)$. Namely, no explicit position dependence is expected within the LDA. Then, the explicit expressions in Eqs. (2.14) can be used.

First, it is shown that for the two body contribution, the gradient corrections dominate over the local term, and hence a local approximation for this term is not accurate. Second, the local approximation of the many body contribution is derived in Eq. (4.12) and it is shown that the gradient corrections do not dominate over the local term, provided that the density varies slowly enough.

In general, the local density approximation of a free energy functional $\mathcal{F}$ and its gradient corrections can be written as, see e.g. Evans (1979),

$$
\mathcal{F}[\rho(\boldsymbol{s})]=\int d A\left(f(\rho(\boldsymbol{s}))+\nabla \rho(\boldsymbol{s}) \cdot \boldsymbol{F}_{2}(\rho(\boldsymbol{s})) \cdot \nabla \rho(\boldsymbol{s})+O\left(\nabla^{4}\right)\right),
$$

where $f(\rho)$ is a scalar-valued function, which gives the free energy density of a homogeneous system with density $\rho$. Furthermore, $\boldsymbol{F}_{2}(\rho)$ is a tensor-valued function given by

$$
\boldsymbol{F}_{2}(\rho)=\left.\frac{-1}{4} \int d A \boldsymbol{s} \otimes \boldsymbol{s} \frac{\delta^{2} \mathcal{F}[\rho(\boldsymbol{s})]}{\delta \rho(\mathbf{0}) \delta \rho(\boldsymbol{s})}\right|_{\rho(\boldsymbol{s})=\rho} .
$$

This expression could be obtained by a Taylor expansion of $\rho(\boldsymbol{s})$ around $\boldsymbol{s}$ and an expansion of $\mathcal{F}[\rho(\boldsymbol{s})]$ in powers of $\nabla$.

It is assumed that it is sensible to make a local approximation, provided that both terms $f(\rho)$ and $\boldsymbol{F}_{2}(\rho)$ are finite, and the gradient correction does not dominate over the first term. The latter sets a lower limit for the typical length scale on which the density profile varies.

\subsubsection{Single slip system}

The system is simplified by only considering one slip system with only two possible Burgers vectors, namely edge dislocations with opposite Burgers vector. Hence without loss of generality, one can say that $\hat{\boldsymbol{\xi}}=\hat{\boldsymbol{z}}$ and $\boldsymbol{b}= \pm b \hat{x}$. Using this simplification, the free energy expression in Eq. (2.20) reads

$$
\begin{aligned}
F\left[\rho_{+}(\boldsymbol{s}), \rho_{-}(\boldsymbol{s}), \boldsymbol{u}_{b}, T\right]= & \frac{1}{2} \int d V \varepsilon_{0}: \mathbb{C}: \boldsymbol{\varepsilon}_{0} \\
& +\frac{1}{2} \int d A d A^{\prime}\left(\rho_{+}(\boldsymbol{s})-\rho_{-}(\boldsymbol{s})\right) u_{\text {edge }}\left(\boldsymbol{s}-\boldsymbol{s}^{\prime}\right)\left(\rho_{+}\left(\boldsymbol{s}^{\prime}\right)-\rho_{-}\left(\boldsymbol{s}^{\prime}\right)\right) \\
& +\int d A\left(\rho_{+}(\boldsymbol{s})-\rho_{-}(\boldsymbol{s})\right) V_{0, \text { edge }}(\boldsymbol{s})+k_{B} T \sum_{+,-} \int d \rho_{ \pm}(\boldsymbol{s})\left(\ln \left(\frac{\rho_{ \pm}(\boldsymbol{s})}{\rho_{ \pm, 0}}\right)-1\right) \\
& +\frac{k_{B} T}{2} \operatorname{Tr}\left[\ln \left(\operatorname{I} \delta\left(\boldsymbol{s}-\boldsymbol{s}^{\prime}\right)+\frac{1}{k_{B} T} \int d z d z^{\prime} G_{0}\left(\boldsymbol{s}-\boldsymbol{s}^{\prime}, z-z^{\prime}\right): \hat{\boldsymbol{\xi}} \otimes \boldsymbol{b} \otimes \hat{\xi} \otimes \boldsymbol{b}\left(\rho_{+}\left(\boldsymbol{s}^{\prime}\right)+\rho_{-}\left(\boldsymbol{s}^{\prime}\right)\right)\right)\right]
\end{aligned}
$$

where $V_{\text {edge, } 0}(\boldsymbol{s}) \equiv V_{b \hat{\boldsymbol{x}}, 0}(\boldsymbol{s})$ is the Peach Koehler potential that the edge dislocations feel, and where $u_{\text {edge }}\left(\boldsymbol{s}-\boldsymbol{s}^{\prime}\right) \equiv u_{b \hat{\boldsymbol{x}}, b \hat{\boldsymbol{x}}}\left(\boldsymbol{s}, \boldsymbol{s}^{\prime}\right)$ is the interaction potential between two equal edge dislocations. It is convenient to use the interaction potential in Fourier space. Using Eqs. (2.14), this reads:

$$
\tilde{u}_{\text {edge }}(\boldsymbol{q})=b^{2} \int d A \int d z d z^{\prime} \hat{\boldsymbol{\xi}} \otimes \hat{\boldsymbol{x}}: \mathrm{G}_{0, i j i, j}\left(\boldsymbol{s}-\boldsymbol{s}^{\prime}, z-z^{\prime}\right): \hat{\boldsymbol{\xi}} \otimes \hat{\boldsymbol{x}} e^{-1 \boldsymbol{q}_{2 D^{\cdot}(\boldsymbol{s}-\boldsymbol{s} \prime)}}=\frac{2 \mu b^{2} L}{1-\nu} \frac{q_{y}^{2}}{q^{4}},
$$

where $q_{y}$ is the $y$-component of $\boldsymbol{q}: q_{y}=\boldsymbol{q} \cdot \hat{\boldsymbol{y}}$, and where $q$ is the length of $\boldsymbol{q}: q=|\boldsymbol{q}|$. The parameter $L$ is the typical persistence length of a dislocation, as introduced in Section 3.2 .

\subsubsection{LDA and gradient corrections of the two body contribution}

In this section, the first and second term in Eq. (4.1) are determined for the two-body contribution. First, the local term is determined from the two-body energy of a homogeneous system with a GND density $\rho_{G N D}$ :

$$
\int d A f_{2 b}\left(\rho_{G N D}\right)=F_{2 b}\left[\rho_{G N D}\right]=\frac{1}{2} \int d A d A^{\prime} \rho_{G N D}^{2} u_{\text {edge }}\left(\boldsymbol{s}-\boldsymbol{s}^{\prime}\right)=\frac{1}{2} \int d A \rho_{G N D}^{2} \tilde{u}_{\text {edge }}\left(\boldsymbol{q}_{2 D}=\mathbf{0}\right)=\left.\frac{\rho_{G N D}^{2}}{2}\left(\frac{2 \mu b^{2} L}{1-\nu} \frac{q_{y}^{2}}{q^{4}}\right)\right|_{\boldsymbol{q}=0} A,
$$

where $A$ is the surface area. The term in parenthesis on the RHS diverges with $q^{-2}$ for small $q$. The smallest wave number in the system in inversely proportional to the largest length scale, which is the system size $R$. The energy density thus diverges as $R^{2}$.

Second, the gradient correction of the two-body contribution is determined using Eq. (4.2): 


$$
\begin{aligned}
\boldsymbol{F}_{2,2 b} & =\frac{-1}{4} \int \mathrm{d} A \boldsymbol{s} \otimes \boldsymbol{s} u_{\text {edge }}(\boldsymbol{s}) \\
& =\left.\frac{1}{4} \int d A \frac{\mathrm{d}}{\mathrm{d} \boldsymbol{q}} \otimes \frac{\mathrm{d}}{\mathrm{d} \boldsymbol{q}}(\exp [-1 \boldsymbol{q} \cdot \boldsymbol{s}])\right|_{\boldsymbol{q}=0} u_{\text {edge }}(\boldsymbol{s}) \\
& =\left.\frac{1}{4} \frac{\mathrm{d}}{\mathrm{d} \boldsymbol{q}} \otimes \frac{\mathrm{d}}{\mathrm{d} \boldsymbol{q}} \tilde{u}_{\text {edge }}\left(\boldsymbol{q}_{2 D}\right)\right|_{\boldsymbol{q}=0} \\
& =\left.\frac{1}{4} \frac{\mathrm{d}}{\mathrm{d} \boldsymbol{q}} \otimes \frac{\mathrm{d}}{\mathrm{d} \boldsymbol{q}} \frac{2 \mu b^{2} L}{1-\nu} \frac{q_{y}^{2}}{q^{4}}\right|_{\boldsymbol{q}=0},
\end{aligned}
$$

where the second equality is just a mathematical identity that is introduced for convenience. The RHS now diverges with $q^{-4}$, and hence it diverges as $R^{4}$ with the system size.

It can thus be concluded that (i) the local contribution is large for large systems and diverges with the system size, and (ii) that the gradient correction dominates over the local contribution when the typical length scale of density fluctuations is smaller than the system size. Therefore, the local density approximation is inaccurate for the two body contribution. This agrees with what was found by Mesarovic (2005).

\subsubsection{LDA and gradient corrections of the many body contribution}

In this section, the local density approximation of the many body term will be determined by calculating the many-body contribution for a homogeneous system with total dislocation density $\rho_{\text {tot }}=\rho_{+}+\rho_{-}$. To this end, first, the eigenvalues of the matrix in the logarithm will be determined, and second, the summation over the logarithm of all eigenvalues will be performed to obtain the trace. This yields the local expression in Eq. (4.12).

The first step is thus to determine the eigenvalues of $1 / k_{B} T \int d z d z^{\prime} G_{0}\left(\boldsymbol{s}-\boldsymbol{s}^{\prime}, z-z^{\prime}\right): \hat{\xi} \otimes \boldsymbol{b} \otimes \hat{\xi} \otimes \boldsymbol{b} \rho_{\text {tot }}$. Note that this tensor is a convolution, and therefore, it can be read as a product in Fourier space. Therefore, it turns out to be more convenient to determine the eigenvalue in Fourier space. The eigenvalue in Fourier space reads

$$
\tilde{\lambda}_{\boldsymbol{U}}\left(\boldsymbol{q}_{2 D}\right) \tilde{\boldsymbol{U}}\left(\boldsymbol{q}_{2 D}\right)=\left(\frac{\rho_{t o t}}{k_{B} T} \mathcal{F}\left[\int d z d z^{\prime} \mathrm{G}_{0}\left(\boldsymbol{s}-\boldsymbol{s}^{\prime}, z-z^{\prime}\right)\right]\left(\boldsymbol{q}_{2 D}\right): \hat{\boldsymbol{\xi}} \otimes \boldsymbol{b}\right)\left(\hat{\boldsymbol{\xi}} \otimes \boldsymbol{b}: \tilde{\boldsymbol{U}}\left(\boldsymbol{q}_{2 D}\right)\right),
$$

where $\boldsymbol{q}_{2 D}$ is the 2D wave-vector. Note that the term in the second bracket on the RHS is a scalar and that the term in the first bracket is a second order tensor independent of $\boldsymbol{U}\left(\mathbf{q}_{2 D}\right)$. This implies that either the eigenvalue is 0 , or that, up to a multiplicative constant, $\tilde{\boldsymbol{U}}\left(\boldsymbol{q}_{2 D}\right)=\tilde{\mathrm{G}}_{0}\left(\boldsymbol{q}_{2 D}\right): \hat{\boldsymbol{\xi}} \otimes \boldsymbol{b}$. Inserting this in Eq. (4.7) implies that the only non-zero eigenvalue is

$$
\lambda(\boldsymbol{q})=\frac{\rho_{\text {tot }}}{k_{B} T} \hat{\boldsymbol{\xi}} \otimes \boldsymbol{b}: \tilde{\mathrm{G}}_{0}\left(\boldsymbol{q}_{2 D}\right): \hat{\boldsymbol{\xi}} \otimes \boldsymbol{b}=\rho_{\text {tot }} \frac{2 \mu b^{2} L}{k_{B} T(1-\nu)} \frac{q_{y}^{2}}{q^{4}} .
$$

The second step is to calculate the many body contribution from these eigenvalues. The logarithm in Eq. (4.3) is the sum of logarithm of the eigenvalues of $\operatorname{I} \delta\left(\boldsymbol{s}-\boldsymbol{s}^{\prime}\right)+\left(1 / k_{B} T\right) \int d z d z^{\prime} \mathrm{G}_{0}\left(\boldsymbol{s}-\boldsymbol{s}^{\prime}, z-z^{\prime}\right): \hat{\boldsymbol{\xi}} \otimes \boldsymbol{b} \otimes \hat{\boldsymbol{\xi}} \otimes \boldsymbol{b} \rho_{\text {tot }}$, which is $1+\lambda(\boldsymbol{q})$ in Fourier space. The trace over the continuous index can be taken by integrating over the wave vector $\boldsymbol{q}_{2 D}$ and by integrating over real space. This yields

$$
F_{m b}\left(\rho_{t o t}, T\right)=\frac{k_{B} T}{2} \int d A \int \frac{d^{2} \boldsymbol{q}_{2 D}}{(2 \pi)^{2}} \ln \left(1+\rho_{t o t} \frac{2 \mu b^{2} L}{k_{B} T(1-\nu)} \frac{q_{y}^{2}}{q^{4}}\right) .
$$

In this explicit form, the integral over $\boldsymbol{q}_{2 D}$ can be performed exactly. For readibility, the shorthand notation $a \equiv \rho_{\text {tot }} 2 \mu b^{2} L / k_{B} T(1-\nu)$ is introduced. Note that $a$ has the dimension of density. Then, using polar coordinates for the integration over $\boldsymbol{q}$, the free energy density as introduced in Eq. (4.1) reads

$$
\begin{aligned}
f_{m b}\left(\rho_{\text {tot }}, T\right) & =\frac{1}{(2 \pi)^{2}} \frac{k_{B} T}{2} \int_{0}^{\infty} d q \int_{0}^{2 \pi} d \phi q \ln \left(1+a \frac{\sin ^{2} \phi}{q^{2}}\right) \\
& =\frac{k_{B} T}{8 \pi^{2}} \int_{0}^{2 \pi} d \phi\left[\frac{q^{2}}{2} \ln \left(1+\frac{a \sin ^{2} \phi}{q^{2}}\right)+\frac{a \sin ^{2} \phi}{2} \ln \left(q^{2}+a \sin ^{2} \phi\right)\right]_{q=0}^{\infty} .
\end{aligned}
$$

At the lower boundary $q=0$, the term in square brackets is equal to $a \sin ^{2} \phi / 2 \ln \left(a \sin ^{2} \phi\right)$, and hence it is finite. However, for large $q$ it diverges. Large values of the wave vector $q$ correspond to small length scales. Integrating over $q$ up to infinity thus corresponds to incorporating phenomena at very small length scales. However, at very small length scales, comparable to the atom spacing in the crystal, we already know that linear elasticity theory breaks down. Hence integrating $q$ up to infinity is physically speaking not admissible, and one should introduce a large cutoff $\Lambda_{0}$ for the integration over $q$, such that 
$\left|\boldsymbol{q}_{2 D}\right| \in\left[0, \Lambda_{0}\right]$. However, physical results should not depend on the exact value of the cutoff $\Lambda_{0}$, since they do not depend on the exact length scale at which elasticity theory breaks down.

It is now assumed that $\Lambda_{0}^{2}$ is much larger than $a$. This yields for the many body contribution:

$$
f_{m b}\left(\rho_{\text {tot }}, T\right)=\frac{k_{B} T}{8 \pi^{2}} \int_{0}^{2 \pi} d \phi \frac{a \sin ^{2} \phi}{2}\left(-\ln \left(\frac{a \sin ^{2} \phi}{\Lambda_{0}^{2}}\right)+1\right) .
$$

The integration over $\phi$ can now be performed straightforwardly using a symbolic toolbox. This finally yields for the free energy density

$$
f_{m b}\left(\rho_{\text {tot }}, T\right)=-\frac{k_{B} T}{8 \pi^{2}} a \ln \left(\frac{a}{4 \Lambda_{0}^{2}}\right)=-\frac{\mu b^{2} L}{8 \pi(1-\nu)} \rho_{\text {tot }} \ln \left(\frac{\rho_{\text {tot }}}{\Lambda^{2}(T)}\right),
$$

where $\Lambda$ is introduced for convenience: $\Lambda^{2} \equiv 4 \Lambda_{0}^{2} k_{B} T(1-\nu) / 2 \mu b^{2} L$. Note that $\Lambda$ has the dimension of inverse length, and that $\Lambda^{2} \gg \rho_{\text {tot }}$, which follows directly from $\Lambda_{0}^{2} \gg a$. This implies that the cutoff length $\Lambda^{-1}$ should be much smaller than the typical dislocation spacing. Hence the many-body contribution is non-zero if $\rho_{\text {tot }}$ is non-zero.

As the free energy density $f_{m b}\left(\rho_{t o t}, T\right)$ depends only on the logarithm of $\Lambda$, all physical quantities are independent of the exact value of the cutoff $\Lambda_{0}$. Namely, if we would take the cutoff twice as large, the free energy would increase by an amount $\mu b^{2} L / 8 \pi(1-\nu) \rho_{\text {tot }} \ln 4$, which is a constant times the density. The constant can thus be interpreted as an additive constant to the chemical potential, which has no physical meaning.

Now, the gradient correction, as introduced in Eq. (4.1), is determined for the many body contribution, see Eq. (4.14) for the result. From this expression, it is then determined under which conditions the gradient correction is small compared to the local contribution, see Eqs. (4.15) and (4.16).

To determine the gradient correction, the second derivative of the free energy with respect to the density profile is needed, see Eq. (4.1). For the many body correction, this derivative is

$$
\begin{aligned}
\left.\frac{\delta^{2} \mathcal{F}_{m b}\left[\rho_{\text {tot }}\left(\boldsymbol{s}^{\prime}\right), T\right]}{\delta \rho_{\text {tot }}(\mathbf{0}) \delta \rho_{\text {tot }}(\boldsymbol{s})}\right|_{\rho_{\text {tot }}(\boldsymbol{s})=\rho_{\text {tot }}}= & -\operatorname{Tr}\left[\left(\operatorname{I} \delta\left(\boldsymbol{s}_{1}-\boldsymbol{s}_{2}\right)+\frac{1}{k_{B} T} \int d z d z^{\prime} \mathrm{G}_{0}\left(\boldsymbol{s}_{1}-\boldsymbol{s}_{2}, z-z^{\prime}\right):: \hat{\xi} \otimes \boldsymbol{b} \otimes \hat{\boldsymbol{\xi}} \otimes \boldsymbol{b} \rho_{\text {tot }}\right)\right. \\
& : \frac{1}{k_{B} T} \int d z d z^{\prime} \mathrm{G}_{0}\left(\boldsymbol{s}_{2}-\boldsymbol{s}_{3}, z-z^{\prime}\right): \hat{\boldsymbol{\xi}} \otimes \boldsymbol{b} \otimes \hat{\boldsymbol{\xi}} \otimes \boldsymbol{b}: \\
& :\left(\operatorname{I} \delta\left(\boldsymbol{s}_{3}-\boldsymbol{s}_{4}\right)+\frac{1}{k_{B} T} \int d z d z^{\prime} \mathrm{G}_{0}\left(\boldsymbol{s}_{3}-\boldsymbol{s}_{4}, z-z^{\prime}\right): \hat{\xi} \otimes \boldsymbol{b} \otimes \hat{\boldsymbol{\xi}} \otimes \boldsymbol{b} \rho_{\text {tot }}\right) \\
& \left.:: \frac{1}{k_{B} T} \int d z d z^{\prime} \mathrm{G}_{0}\left(\boldsymbol{s}_{4}-\boldsymbol{s}_{1}, z-z^{\prime}\right): \hat{\boldsymbol{\xi}} \otimes \boldsymbol{b} \otimes \hat{\boldsymbol{\xi}} \otimes \boldsymbol{b}\right] \delta\left(\boldsymbol{s}_{1}\right) \delta\left(\boldsymbol{s}_{3}-\boldsymbol{s}\right) .
\end{aligned}
$$

This is again a convolution, which results in a product in Fourier space. This can be expressed in the eigenvalues $\lambda(\boldsymbol{q})$ from the previous section. Again, the integration over the wave vector can be performed using a symbolic toolbox. Details of this derivation can be found in Appendix B. This yields for the gradient correction:

$$
\boldsymbol{F}_{2, m B}\left(\rho_{\text {tot }}, T\right)=\frac{1}{24 \pi} \frac{k_{B} T}{\rho_{\text {tot }}^{2}}\left(\begin{array}{ll}
1 & 0 \\
0 & -1+\frac{3 \sqrt{\rho_{\text {tot }}} R}{8} \sqrt{\frac{2 \mu b^{2} L}{k_{B} T(1-\nu)}}
\end{array}\right),
$$

where $R$ is the system size. Derivatives in the $x$-direction do therefore not dominate over the local term provided that

$$
\begin{aligned}
& \frac{1}{24 \pi} \frac{k_{B} T}{\rho_{\text {tot }}^{2}}\left|\partial_{\chi} \rho_{\text {tot }}(\boldsymbol{s})\right|^{2} \ll \frac{\mu b^{2} L}{8 \pi(1-\nu)} \rho_{\text {tot }}\left|\ln \left(\frac{\rho_{\text {tot }}}{\Lambda^{2}(T)}\right)\right| \\
& \Rightarrow\left(\frac{\left|\partial_{x} \rho_{\text {tot }}(\boldsymbol{s})\right|}{\rho_{\text {tot }}}\right)^{2} \ll 3 \frac{\Xi}{1-\nu} \rho_{\text {tot }}\left|\ln \left(\frac{\rho_{\text {tot }}}{\Lambda^{2}(T)}\right)\right| \text {, }
\end{aligned}
$$

where the parameter $\Xi$ is defined in Section 3.2, and it is typically much larger than 1. Moreover, $\left|\ln \left(\rho_{\text {tot }} / \Lambda^{2}(T)\right)\right|$ is much larger than one, as $\Lambda^{2} \gg \rho_{\text {tot }}$.

It can thus be concluded that the LDA is valid if the typical length scale of variations of the total dislocation density in $x$ direction, $\rho_{\text {tot }}|| \partial_{x} \rho_{\text {tot }}$, is much longer than the average dislocation spacing $1 / \sqrt{\rho_{\text {tot }}}$. As both $\Xi$ and $\left|\ln \left(\rho_{\text {tot }} \mid \Lambda^{2}(T)\right)\right|$ are large, the condition in Eq. (4.15) is not very restrictive.

However, when the total dislocation density varies in the $y$-direction, the gradient does not dominate over the local term 
provided that

$$
\begin{gathered}
\frac{1}{64 \pi} \frac{k_{B} T}{\rho_{\text {tot }}^{3 / 2}} R \sqrt{\frac{2 \mu b^{2} L}{k_{B} T(1-\nu)}}\left|\partial_{y} \rho_{\text {tot }}(\boldsymbol{s})\right|^{2} \ll \frac{\mu b^{2} L}{8 \pi(1-\nu)} \rho_{\text {tot }}\left|\ln \left(\frac{\rho_{\text {tot }}}{\Lambda^{2}(T)}\right)\right| \\
\Rightarrow \quad\left(\frac{\left|\partial_{y} \rho_{\text {tot }}(\boldsymbol{s})\right|}{\rho_{\text {tot }}}\right)^{2} \ll 4 \sqrt{\frac{2 \Xi}{1-\nu}} \frac{\sqrt{\rho_{\text {tot }}}}{R}\left|\ln \left(\frac{\rho_{\text {tot }}}{\Lambda^{2}(T)}\right)\right| .
\end{gathered}
$$

The LDA is thus valid if the typical length scale of variations in the $y$-direction, $\rho_{\text {tot }} / \mid \partial_{y} \rho_{\text {tot }} l$, is much larger than $\sqrt{R / \sqrt{\rho_{\text {tot }}}}$, which is much larger than the average dislocation spacing. If the system is infinitely large, Eq. (4.16) implies that no variations in the $y$-direction are allowed. Furthermore, the RHS of Eq. (4.16) is only inversely proportional to the square-root of $\Xi$. Therefore, the condition in Eq. (4.16) is much more restrictive than in Eq. (4.15).

The difference between variations in $x$ - and $y$-direction originates from the difference in screening in both directions. As can be seen in Fig. 4, in the $x$-direction, dislocations with opposite Burgers vector are attracted. These dislocations screen the effect of the dislocation at the origin. However, in the $y$-direction, dislocations with equal Burgers vector are attracted. These dislocations enhance the effect of the dislocation at the origin.

The restriction on the variations in density in the $y$-direction also restricts the possible choice for the glide-plane distribution. Namely, if a dislocation density profile is considered which is only nonzero at discrete glide-planes, this implies that the derivative of the density in $y$-direction is large, and hence that the condition in Eq. (4.16) is violated. Therefore, only profiles that are more or less homogeneous in the $y$-direction are allowed for. This means that the glide planes are smearedout.

\subsubsection{Conclusions about the LDA and comparison to literature}

It was found in this section that the LDA is applicable for the many-body contribution, provided that the density profile varies slow enough, but that the LDA is not applicable for the two body contribution. As in Eq. (2.22), the background, twobody and loading contributions can be combined into the elastic contribution. This results in the following semi-local free energy expression:

$$
\begin{aligned}
F_{L D A}\left[\rho_{+}(\boldsymbol{s}), \rho_{-}(\boldsymbol{s}), \boldsymbol{u}_{b}, T\right]= & \frac{1}{2} \int d V \boldsymbol{\varepsilon}_{\mathrm{mf}}: \mathbb{C}: \boldsymbol{\varepsilon}_{\mathrm{mf}}+k_{B} T \sum_{+,-} \int d A \rho_{ \pm}(\boldsymbol{s})\left(\ln \left(\frac{\rho_{ \pm}(\boldsymbol{s})}{\rho_{ \pm, 0}}\right)-1\right) \\
& -\frac{\mu b^{2} L}{8 \pi(1-\nu)} \int d A\left(\rho_{+}(\boldsymbol{s})+\rho_{-}(\boldsymbol{s})\right) \ln \left(\frac{\rho_{+}(\boldsymbol{s})+\rho_{-}(\boldsymbol{s})}{\Lambda^{2}}\right) .
\end{aligned}
$$

This expression might seem local at first glance, as it is written as a single spatial integral. However, one should realize that the free energy is not to be interpreted as a functional of the mean field strain $\boldsymbol{\varepsilon}_{\mathrm{mf}}$, but rather of the dislocation density profiles $\rho_{+}(\boldsymbol{s})$ and $\rho_{-}(\boldsymbol{s})$, and the displacement $\boldsymbol{u}_{b}$ of the boundary. The mean field strain itself is a strongly non-local functional of the dislocation density, see Eq. (2.21).

In the work of Mesarovic (2005), it was also shown that the two-body interaction energy cannot be approximated by a

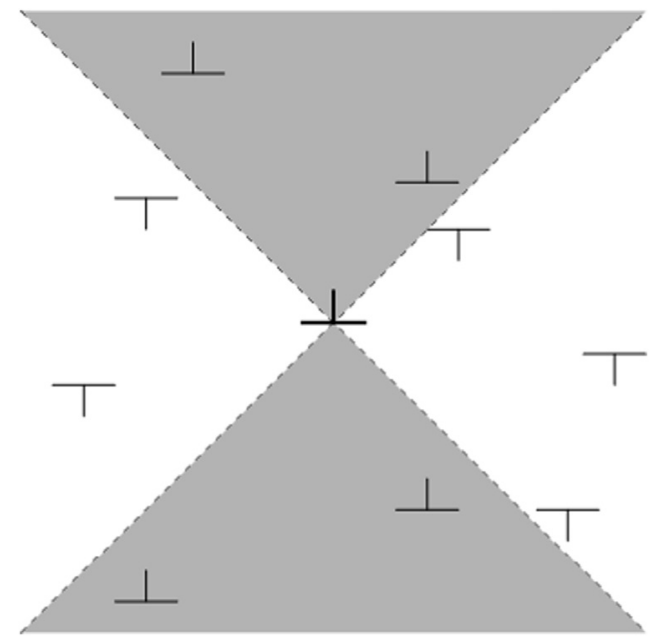

Fig. 4. In the white regions, edge dislocations with opposite Burgers vector are attracted by the dislocation in the origin, and thereby they screen the effect of the central dislocation. In the shaded regions, edge dislocations with equal Burgers vector are attracted, which strengthens the effect of the central dislocation. 
local expression. Moreover, in later work (Mesarovic et al., 2010), it was shown that the contribution due to coarsening can be approximated by a local form provided that the typical length over which the dislocation density profile varies is much longer than the average dislocation spacing. This agrees with the explicit expression obtained here.

In the work of Groma et al. (2014), it was argued that no new internal length scale should arise from coarse-graining, as the interaction between dislocations is scale-free. The free energy obtained here satisfies this constraint, as the many-body contribution depends only logarithmically on the cutoff $\Lambda$.

The free energy of a system with spatially constant dislocation density was determined by Mizushima (1960), Ninomiya (1978), Yamamoto and Izuyama (1988) and Burakovsky et al. (2000). The systems considered in their work do not have a net dislocation content, and the free energy expressions were used to explain the melting transition of crystals. The suggested expressions all entail a $-\rho \ln \rho$-term with a prefactor proportional to the shear modulus and the Burgers vector squared, but independent of temperature, which is analogous to the expression for $F_{m b}$ found here.

Additional terms proportional to $\rho, \rho^{3 / 2}$ and $\rho^{2}$ were suggested in Mizushima (1960), Ninomiya (1978), Yamamoto and Izuyama (1988), Burakovsky et al. (2000). The terms linear in the density result from the energy of individual dislocation lines. In our study, self-energy contributions are neglected, and hence the linear contributions are absorbed in the chemical potential. The term proportional to $\rho^{3 / 2}$ results from the configurational entropy of dislocation networks. This term is not found in our work, as joggs and connections between dislocations do not occur in systems with only straight and parallel dislocations. The term quadratic in the density in Yamamoto and Izuyama (1988) resulted from a non-overlapping condition for dislocations. This was not accounted for in our analysis, and hence such a term is not found.

In the work of Svendsen, Klusemann et al. (2012), Bargmann and Svendsen (2012), Svendsen (2002), the free energy is separated in a linear elastic part, and another contribution that is written as a power series in the GND and SSD density separately. In later work (Svendsen and Bargmann, 2010), forms other than polynomials were also considered for this second term. Here it was shown that a local expression is indeed possible for the many body contribution, and a series expansion of the local expression obtained here could be valid in a certain regime.

In the work of Panyukov and Rabin (1999), the free energy of a system of dislocation loops was determined. In that work, the density of dislocations was assumed to be independent of the Burgers vector, and hence no net dislocation was considered, both locally and globally. Despite the differences in problem setup with our analysis, the free energy expression found in Panyukov and Rabin (1999, Eq. (65)) is remarkably similar to what we found in Eq. (4.9). As in our work, only straight and infinitely long dislocations are considered, it was possible to perform the integration over the wave vector in Eq. (4.9) explicitly, which was impossible in the work of Panyukov and Rabin (1999).

\subsection{Zero temperature}

In this work, the coarse-graining was performed at non-zero temperature, and the temperature dependence in Eq. (2.22) is explicit. On the contrary, in literature, the temperature dependence is often not clear, as the system is considered at isothermal conditions. Sometimes, an explicit split is made between energetic and entropic contributions, for example in Groma et al. (2006, 2007). In this section, we determined the zero temperature limit of our free energy expression, to see what the effect of working at non-zero temperature is. The result will be compared to literature.

To study the zero temperature limit of the free energy expression in Eq. (2.22), it is first noted that the mean field elastic contribution $F_{\text {elas }}$ as defined in Eq. (3.1), is independent of the temperature, and hence does not change in the limit.

Second, the statistical contribution defined in Eq. (3.3) is proportional to the temperature. Therefore, it vanishes in the zero temperature $\operatorname{limit}_{\lim _{T \rightarrow 0}} F_{\text {stat }}=0$. And finally, the many body contribution as defined in Eq. (3.4) is considered. This term can be written as

$$
F_{m b}=\frac{k_{B} T}{2} \int d A \sum_{m=1}^{9} \ln \left(1+\frac{1}{k_{B} T} \lambda_{m}(\boldsymbol{s})\right)
$$

where $\lambda_{m}(\boldsymbol{s})$ is the $m^{\text {th }}$ eigenvalue of the matrix $\sum_{\boldsymbol{b}} \int d z d z^{\prime} \mathrm{G}_{0}\left(\boldsymbol{s}, z ; \boldsymbol{s}^{\prime}, z^{\prime}\right): \rho_{\boldsymbol{b}}\left(\boldsymbol{s}^{\prime}\right) \hat{\boldsymbol{\xi}} \otimes \boldsymbol{b} \otimes \hat{\xi} \otimes \boldsymbol{b}$, and the sum runs over all eigenvalues. As $\mathrm{G}_{0}\left(\boldsymbol{s}, z ; \boldsymbol{s}^{\prime}, z^{\prime}\right)$ is independent of the temperature $T$, the eigenvalues $\lambda_{m}(\boldsymbol{s})$ are independent of temperature as well. Thus, in the zero temperature limit, the many body contribution reads

$$
\lim _{T \rightarrow 0} F_{m b}=\int d A \sum_{m=1}^{9} \lim _{T \rightarrow 0} \frac{k_{B} T}{2} \ln \left(1+\frac{1}{k_{B} T} \lambda_{m}(\boldsymbol{s})\right)=\int d A \sum_{m=1}^{9} 0=0,
$$

and hence vanishes.

At first glance, this result is contradictory with the explicit result that was obtained for a spatially homogeneous dislocation density in Eq. (4.12). Namely, that many body contribution is non-zero when the dislocation density is non-zero. However, in the derivation, it was used just before Eq. (4.12) that one can choose a cutoff wave number $\Lambda_{0}$ such that $\Lambda_{0}^{2} \gg \rho_{\text {tot }} 2 \mu b^{2} L / k_{B} T(1-\nu)$, which is clearly impossible in the zero temperature limit.

Hence once the LDA is made at finite temperature, the zero temperature limit cannot be taken anymore. Other approximation techniques for the free energy might also involve additional assumptions on the temperature. Therefore, we think that in general one should be careful in taking the zero temperature limit when approximations are made. 
So to conclude, the free energy at $T=0$ can be expressed as

$$
F\left[\rho_{\boldsymbol{b}}(\boldsymbol{s}), \boldsymbol{u}_{b}, T=0\right]=F_{\text {elas }}\left[\rho_{\boldsymbol{b}}(\boldsymbol{s}), \boldsymbol{u}_{b}\right]=F_{\text {background }}\left[\boldsymbol{u}_{b}\right]+F_{2 \mathrm{~b}}\left[\rho_{\boldsymbol{b}}(\boldsymbol{s})\right]+F_{\text {loading }}\left[\rho_{\boldsymbol{b}}(\boldsymbol{s}), \boldsymbol{u}_{b}\right] .
$$

It is tempting to interpret the free energy at zero temperature as the internal energy $U$ of the system, as $F=U-T S$. However, the internal energy should formally be determined by first calculating the entropy from $S=-\delta F / \delta T$ and subsequently using the relation $U=F+T S$. When this is done using the free energy expression in Eq. (2.22), it turns out that the internal energy $U$ differs from (4.20) by a contribution that vanishes only at zero temperature. Therefore, the internal energy $U$ at $T>0$ is not given by the free energy at zero temperature.

It was recognized in the work of Groma et al. (2006, 2007) and Limkumnerd and Van der Giessen (2008) that the physical temperature of the system is close to zero, which means that the statistical contribution $F_{\text {stat }}$ is much smaller than the energetic contribution. Hence it was concluded that it plays no role. However, another 'phenomenological temperature' was introduced to cover many body effects. In our work here, it was indeed found that many body effects vanish at zero temperature. However, at small but finite temperature, many body effects appear naturally and in a scale-free form, as was anticipated in Groma et al. (2014).

In the crystal plasticity model of Bammann (2001), the temperature dependence is explicit. Three important differences with our model can be distinguished. First, the elastic constants depend on temperature. In our work, this dependence was not put in the model on the microscopic level, and therefore, it is not present in the result either. An extension to temperature dependence on the microscopic level could be made. Second, in the work of Bammann, an energy contribution is found that depends only on temperature, and not on the strain of the material. This energy contribution is perhaps related to the phonon energy in the crystal. The mechanical response of the system was found to be independent of this energy contribution. In the work presented here, the focus was on deriving the free energy due to mechanical loading. Therefore, phonon energy was interpreted as an irrelevant constant contribution, and was therefore neglected. Third, the free energy expression obtained here comprises an statistical and a many body contribution that depends both on temperature and on dislocation density. Comparable terms were not proposed by Bammann. This is probably because these contributions are due to the effect of non-zero temperature on dislocation distributions, rather than only the effect of the temperature on the lattice.

\subsubsection{Equally spaced glide planes}

The free energy at zero temperature can be further simplified for dislocations on equally spaced glide planes. As shown in several papers (Scardia et al., 2014; Geers et al., 2013; Baskaran et al., 2010), this geometry simplifies the mathematical analysis of the problem considerably and the coarse graining can be done using $\Gamma$-convergence, see Scardia et al. (2014), Geers et al. (2013). In this section, the free energy at zero temperature in Eq. (4.20) is evaluated in this special geometry, and the results are compared with those obtained using $\Gamma$-convergence. This case can be considered as an example in which the discrete glide planes are still resolved on the macroscopic level.

The exact geometry that is used is depicted in Fig. 5(a). The glide planes are oriented in $\hat{\boldsymbol{x}}$-direction and the dislocations pile up against an infinitely long, vertical wall at $x=0$. Note that only glide planes with dislocations are considered, and that planes without dislocations are not accounted for. The glide plane spacing is $h$, and, as in Scardia et al. (2014), Geers et al. (2013), only positive dislocations are considered. Moreover, it is assumed that the loading is the same on each glide plane and hence $\varepsilon_{0}$ and $V_{\text {edge }}$ are both only functions of $x$.

Because of the symmetry of the system and loading under a translation over a distance $h$ in $\hat{\boldsymbol{y}}$-direction, the density of dislocations in the glide plane is independent of the $y$-coordinate of the glide plane. Therefore, the density of dislocations can be written as

$$
\rho_{+}(\boldsymbol{s})=\rho(x) \sum_{n=-\infty}^{\infty} \delta(y-h n),
$$

where the summation runs over all integers, and where the delta function indicates the positions of the glide planes as in Fig. 3. Hence, the density is zero if there is no glide plane, and $\rho(x)$ in the glide plane. Note that both $\delta(y-h n)$ and $\rho(x)$ have the dimension of inverse length. The background energy was not accounted for in Scardia et al. (2014), Geers et al. (2013), and will thus be left out here as well. Using Eq. (4.21) in Eq. (4.20) for the free energy at zero temperature yields

$$
\begin{aligned}
F\left[\rho_{\boldsymbol{b}}(\boldsymbol{s}), \boldsymbol{u}_{b}, T\right. & =0] \\
& =\int \mathrm{dA} \rho(x) \sum_{n=-\infty}^{\infty} \delta(y-h n) V_{\text {edge }}(x)+\frac{1}{2} \int \operatorname{dAdA}^{\prime} \rho(x) \sum_{n=-\infty}^{\infty} \delta(y-\mathrm{hn}) u_{\boldsymbol{b}, \boldsymbol{b}^{\prime}}\left(\boldsymbol{s}, \boldsymbol{s}^{\prime}\right) \rho\left(x^{\prime}\right) \sum_{n^{\prime}=-\infty}^{\infty} \delta\left(y^{\prime}-\mathrm{hn}^{\prime}\right) .
\end{aligned}
$$

This expression can be rewritten by performing the summations over $n$ and the integrations over $y$ and $y^{\prime}$. The energy can then be expressed in terms of the number of glide planes $N_{y}$ and the interaction energy of a regular wall of dislocations with a single dislocations, $u_{\text {wall }}(\Delta x)$, which reads 

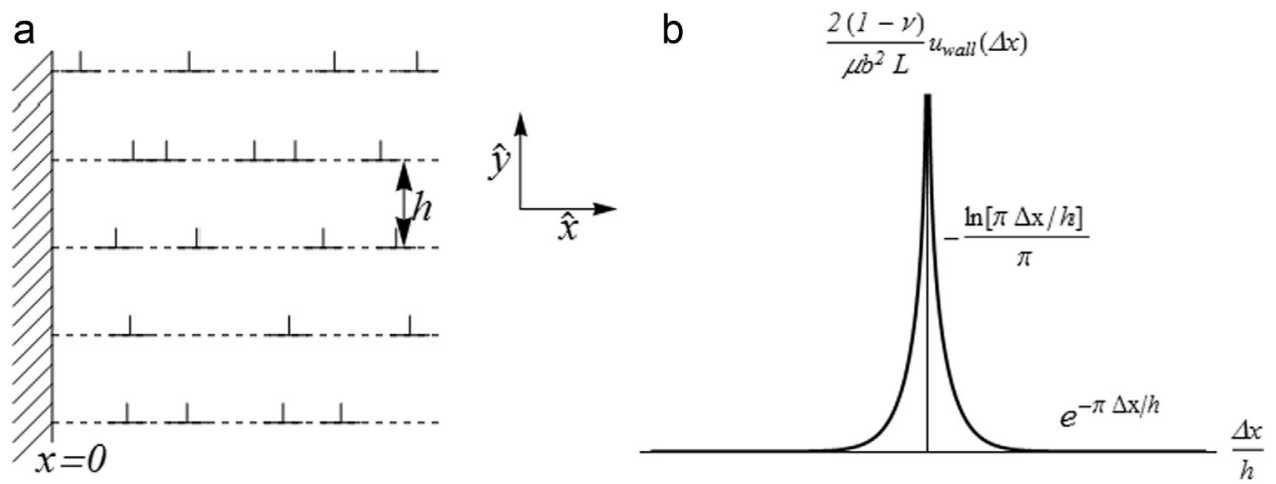

Fig. 5. The geometry studied in this section: positive dislocations on equally spaced glide planes that pile-up against a wall at $x=0$ (fig. (a)), and the interaction potential between two dislocation walls, as in Eq. (4.23) (fig. (b)).

$$
u_{\text {wall }}(\Delta x)=\frac{\mu b^{2} L}{2(1-\nu)}\left(\frac{\Delta x}{h} \operatorname{coth}\left(\pi \frac{\Delta x}{h}\right)-\pi^{-1} \ln \left(2 \sinh \left(\pi \frac{\Delta x}{h}\right)\right)\right)
$$

as shown in Appendix C. The potential $u_{\text {wall }}(\Delta x)$ is plotted in Fig. 5(b). The free energy per glide plane thus reads

$$
\frac{F\left[\rho_{\boldsymbol{b}}(\boldsymbol{s}), \boldsymbol{u}_{b}, T=0\right]}{N_{y}}=\int d x \rho(x) V_{\mathrm{edge}}(x)+\frac{1}{2} \int d x d x^{\prime} \rho(x) u_{\text {wall }}\left(x-x^{\prime}\right) \rho\left(x^{\prime}\right) .
$$

As shown in Fig. 5(b), the interaction potential is not long-ranged anymore, as opposed to the original interaction potential $u_{\text {edge }}$. Therefore, the two body term can be approximated by a local expression as in Eq. (4.1), as long as the length scale associated with density fluctuations is small enough. This is clear by considering the LDA of Eq. (4.24) explicitly using the expressions in Eqs. (4.1) and (4.2). The free energy density of the homogeneous system reads

$$
\frac{f_{2 b}(\rho)}{N_{y}}=\rho^{2} 2 \int_{0}^{\infty} d x-x^{\prime} u_{\text {wall }}\left(x-x^{\prime}\right)=\frac{\mu b^{2} L}{2(1-\nu)} \rho^{2} \frac{h}{3},
$$

and the gradient correction term reads

$$
\frac{F_{2,2 b}}{N_{y}}=\frac{-1}{4} 2 \int_{0}^{\infty} d x x^{2} u_{\text {wall }}(x)=\frac{-1}{4} \frac{\mu b^{2} L}{2 \pi(1-\nu)} \frac{h^{3} \pi}{45},
$$

where both integrals were evaluated straightforwardly using a symbolic toolbox. The gradient correction is thus much smaller than the local contribution as long as

$$
\frac{\pi h}{3} \rho^{2} \gg \frac{h^{3} \pi}{180}\left|\rho^{\prime}(x)\right|^{2} \Rightarrow \frac{60}{h^{2}} \gg\left(\frac{\left|\rho^{\prime}(x)\right|}{\rho}\right)^{2},
$$

so when the typical length scale of density changes $\rho / \rho^{\prime}(x) \mid$ is much larger than the glide plane spacing $h$. The energy in Eq. (4.22) can thus be rewritten as

$$
\frac{F\left[\rho_{\boldsymbol{b}}(\boldsymbol{s}), \boldsymbol{u}_{b}, T=0\right]}{N_{y}}= \begin{cases}\int d x \rho(x) V_{\text {edge }}(x)+\frac{h}{12} \frac{\mu b^{2} L}{(1-\nu)} \rho(x)^{2} & \text { if }\left(\frac{\left|\rho^{\prime}(x)\right|}{\rho}\right)^{2} \ll \frac{60}{h^{2}} \\ \int d x \rho(x) V_{\text {edge }}(x)+\frac{1}{2} \int d x d x^{\prime} \rho(x) \rho\left(x^{\prime}\right) u_{\text {wall }}\left(x-x^{\prime}\right) & \text { else. }\end{cases}
$$

Now, the result in Eq. (4.28) is compared to what was found in Scardia et al. (2014), Geers et al. (2013). In these papers, three parameter regimes were distinguished in which different asymptotic forms of the energy expression were found. The exact separation of these regimes depend on the details of the coarse-graining method, and can thus not be compared with the result obtained here. However, in the three regimes good agreement with the expression in Eq. (4.28) is found.

In the so-called subcritical parameter regime, the glide plane spacing is large compared to the typical thickness of the pile-up. Therefore, the influence of different glide planes on each other is assumed to be negligible in Scardia et al. (2014), Geers et al. (2013), and hence it is the "single glide plane regime". The scaling implies that the second case in Eq. (4.28) should be considered. The interaction energy $u_{\text {wall }}\left(x-x^{\prime}\right)$ is logarithmic at small distances, and hence the obtained expression is equivalent to Eq. (24) in Scardia et al. (2014).

In the intermediate parameter regime, the thickness of the pile-up is much longer than the glide plane spacing, but the typical distance between dislocations in $x$-direction is smaller than $h$. This implies that the first case in Eq. (4.28) should be 
considered. And indeed, this energy expression is equivalent to Eq. (40) in Scardia et al. (2014).

In the supercritical parameter regime, the typical distance between dislocations in $x$-direction is larger than $h$. This implies again that the second case in Eq. (4.28) should be considered. To study the extreme case, the limit $h \rightarrow 0$ should be taken. The result of this limit depends on the behavior of $\rho(x)$ in this limit. If $\rho(x)$ increases slower than $h^{-1 / 2}$ if $h$ goes to 0 , the second term in Eq. (4.28) vanishes. On the contrary, if $\rho(x)$ increases faster than $h^{-1 / 2}$, the second term blows up and dominates the integral. This extreme case is what was found in Scardia et al. (2014), Geers et al. (2013). However, the behavior under the limit $h \rightarrow 0$ is only a mathematical limit that cannot be achieved in an experimental setting.

An important difference between the coarse-graining using $\Gamma$-convergence, as performed in Scardia et al. (2014), Geers et al. (2013), and the method employed in this work is that for the former, dislocations align in so-called tilt walls. This means that the discrete dislocation density is the same for all glide planes. In the latter procedure presented here, it is only assumed after coarse-graining that the average density of dislocations is independent of the glide plane. It is remarkable that despite this difference between the two coarse-graining methods, the resulting energy expressions are equivalent.

\section{Implication for crystal plasticity}

The free energy plays a crucial role in most crystal plasticity frameworks. In this section, the implications for crystal plasticity that follow from the free energy derivation presented in this paper are presented. For clarity, our work is only compared with Gurtin (2008).

The free energy used in the work of Gurtin is "given by standard elastic strain energy, augmented by a defect energy $\Psi(\rho)$ ", where $\rho=\left\{\rho_{\mathrm{GND}, \mathrm{e}}^{1}, \ldots, \rho_{\mathrm{GND}, \mathrm{e}}^{N}, \rho_{\mathrm{GND}, \mathrm{s}}^{1}, \ldots, \rho_{\mathrm{GND}, \mathrm{s}}^{N}\right\}$ with $\rho_{\mathrm{GND}, \mathrm{e}}^{i}$ and $\rho_{\mathrm{GND}, \mathrm{s}}^{i}$ the densities of geometrically necessary edge and screw dislocations on slip system $i$, respectively. The free energy density thus reads

$$
\psi=\frac{1}{2} \boldsymbol{E}^{e}: \mathbb{C} \boldsymbol{E}^{e}+\Psi(\rho)
$$

The derivative of the defect energy with respect to the dislocation densities are used as a closure for the dynamic equations. These derivatives are referred to as the energetic defect forces.

The split between standard (mean field) elastic energy and a local contribution that only depends on dislocation density, can also be recognized in our result in Eq. (4.12). The defect energy for a single slip system with only edge dislocations that follows from Eq. (4.12), reads

$$
\begin{aligned}
\Psi\left(\rho_{+}, \rho_{-}\right) & =k_{B} T \sum_{+,-} \rho_{ \pm}\left(\ln \left(\frac{\rho_{ \pm}}{\rho_{ \pm, 0}}\right)-1\right)-\frac{\mu b^{2} L}{8 \pi(1-\nu)}\left(\rho_{+}+\rho_{-}\right) \ln \left(\frac{\rho_{+}+\rho_{-}}{\Lambda^{2}}\right) \\
& =k_{B} T \sum_{+,-} \frac{\rho_{\text {tot }} \pm \rho_{\mathrm{GND}}}{2}\left(\ln \left(\frac{\rho_{\mathrm{tot}} \pm \rho_{\mathrm{GND}}}{2 \rho_{ \pm, 0}}\right)-1\right)-\frac{\mu b^{2} L}{8 \pi(1-\nu)}\left(\rho_{\mathrm{tot}}\right) \ln \left(\frac{\rho_{\mathrm{tot}}}{\Lambda^{2}}\right),
\end{aligned}
$$

where $\rho_{\text {tot }}=\rho_{+}+\rho_{-}$and $\rho_{\mathrm{GND}}=\rho_{+}-\rho_{-}$are the total and GND density, respectively. The energetic defect forces are then different for positive and negative dislocations and read:

$$
f_{ \pm}\left(\rho_{+}, \rho_{-}\right) \equiv \frac{\partial \Psi\left(\rho_{+}, \rho_{-}\right)}{\partial \rho_{ \pm}}=k_{B} T \ln \left(\frac{\rho_{ \pm}}{\rho_{ \pm, 0}}\right)-\frac{\mu b^{2} L}{8 \pi(1-\nu)}\left(\ln \left(\frac{\rho_{\text {tot }}}{\Lambda^{2}}\right)+1\right) .
$$

The striking difference between Eqs. (5.1) and (5.2) is that in the former, the defect energy only depends on GND densities, while in the latter, the defect energy depends on both positive and negative density independently. To use the free energy expression obtained here, the modeling as performed in Gurtin (2008) should thus be extended to positive and negative densities separately.

At first sight, it is remarkable that the macroscopic free energy depends also on the total dislocation density, and not only on the GND density, whereas the driving force on microscopic level, i.e. the Peach-Koehler force, can be written in terms of GNDs only. However, the total density appears in the many body contribution, which is the screening correction to the leading order mean field contribution, see the discussion in Section 3.1. Screening arises as a dislocation influences its environment, i.e. it is more likely to find positive dislocations in the immediate neighborhood of a negative dislocation, than to find negative ones. Screening effects usually depend on the total dislocation density, and not on the GND density, see e.g. Limkumnerd and Van der Giessen (2008). On the microscopic level there is no screening, as the positions of the dislocations are prescribed.

So to conclude, the research presented here implies that for a single slip system, a crystal plasticity model should incorporate the density and current of both positive and negative dislocations, and not just the net density. It is expected that the same holds for multiple slip systems. It is however beyond the scope of this research to decide whether it is more appropriate in a dynamical framework to use the total and GND density of dislocations for each slip system as a modeling variable, or to use densities of dislocations with different Burgers vectors.

Previously, it was indicated by Svendsen and Bargmann (2010) that thermodynamically consistent crystal plasticity 
models based on the free energy are not restricted to the use of GND densities only. However, most models treated in that work use either only the GND density (Kuroda and Tvergaard, 2008; Evers, 2004; Evers et al., 2004; Bayley et al., 2007; Ertürk et al., 2009) or use a form of the free energy that can be split additively into a part depending on the GND or SSD density Ekh et al. (2007).

Another difference between this work and Gurtin (2008), is that here, it was shown that the full free energy expression in Eq. (4.12) is strongly non-local, and hence that local forms of thermodynamic laws do not apply. On the contrary, in the work of Gurtin (2008), a local form of the second law, the local free energy imbalance, is used.

\section{Summary and discussion}

In this work, an explicit expression is derived for the free energy of a coarse-grained dislocation density profile in a finite medium, subjected to a prescribed boundary deformation. The free energy was determined by using statistical mechanics tools to average over many microscopic realizations of the system to retrieve the coarse-grained system properties.

The main result of this paper is the free energy expression in Eq. (2.22). This has been obtained from the grand-canonical partition function, derived in our earlier work (Kooiman et al., 2014). In the present paper, a Legendre transform to the canonical ensemble is performed, which results in the free energy expression in terms of the dislocation density, Eq. (2.22). The obtained expression is an explicit functional of the dislocation density profile, the mechanical loading at the boundary and the temperature. The free energy depends on the finite volume as the elastic energy density is only integrated over a finite volume, and as the interaction between dislocations is determined in a finite medium.

The free energy in Eq. (2.22) comprises three terms: the mean field elastic energy, the statistical contribution of the dislocations and the many-body contribution that results from the local arrangement of dislocations. Explicit expressions for these three terms arise naturally from the coarse-graining procedure.

For the statistical and many-body contribution, the local density approximation (LDA) can be used to obtain the semilocal expression in Eq. (4.12) in terms of the dislocation density, provided that the density profile does not vary too much on small length scales (see Section 4.1 .3 for a detailed discussion). These two contributions together form the so-called defect energy, used in crystal plasticity models. From this, explicit expressions for the defect forces were derived, see Eq. (5.3).

The most prominent difference between the defect energy from most crystal plasticity models and the defect energy derived here, is that the former depends usually only on GND densities, while the latter depends on the density of both positive and negative dislocations separately. This implies that a microscopically based crystal plasticity model should incorporate the density and current of both positive and negative dislocations for slip system, rather than the GND-related quantities only.

Furthermore, it was shown that the statistical and many-body contribution vanish at zero temperature. However, at low but finite temperature, the many-body contribution cannot be neglected.

The energy at zero temperature is simplified further for a system with equally spaced glide planes. In this specific case, the coarse-graining was also done using $\Gamma$-convergence in the literature. It is concluded here that coarse-graining using statistical physics yields the same result as $\Gamma$-convergence.

The free energy was derived by systematic coarse-graining of microstates, characterized by a finite volume subjected to given boundary deformations which contains discrete dislocations with different possible Burgers vectors $\boldsymbol{b}$ on discrete glide planes. The averaging is then performed over all possible numbers and positions of dislocations, and over all strain fields that match the incompatibility imposed by the dislocations and the applied deformation at the boundary.

The main approximation in the derivation is that the system is far away from criticality. This implies that the obtained free energy expression is only accurate far away from transition points. Examples of such points are the unbinding transition of dislocations and the transition from elastic to plastic material response.

So to conclude, the new and innovative aspects of the free energy expression in Eq. (2.22) are the following:

- The free energy expression originates from statistically averaging straight and parallel, discrete dislocations in a finite volume. Apart from the straight and parallel nature of the dislocations, no assumptions on the dislocation arrangement were made.

- Properties that have proven to be important for the mechanical behavior have been included, namely the presence of glide planes and the finite volume.

- In several special cases the free energy expression reduces to a simple expression that can be implemented efficiently in a numerical scheme. The special cases considered here are a local density approximation for a single slip system, the zero temperature limit, and equally spaced glide planes.

- This free energy expression yields microscopically derived expressions for energetic defect forces in crystal plasticity models (Gurtin, 2008). Moreover, the derivation implies that crystal plasticity models can better be formulated in terms of densities and currents of both positive and negative dislocations on each slip system, rather than in terms of GNDrelated quantities only.

For future research, it would be interesting to weaken the limitations as described in Section 3.3. This can be done by 
replacing the Gaussian approximation by a more accurate one, for example the Villain approximation (Kleinert, 1989). Then, the free energy expression could also be used to study the melting transition, as was also studied by Mizushima (1960), Ninomiya (1978), Yamamoto and Izuyama (1988) and Burakovsky et al. (2000).

Besides that, it may be possible to derive along the same lines a free energy expression for dislocations that are not straight and not parallel. Such an extended phase space was also argued for by Hochrainer et al. (2014). A comparable analysis for a homogeneous system of curved dislocations was performed before by Panyukov and Rabin (1999).

Furthermore, new simple expressions can be obtained by considering other special cases. For example, the local density approximation can be determined for multiple slip systems. From such an expression and the resulting dislocation density profiles, the importance of the different slip systems and their mutual influence can be determined.

As we have shown that the zero temperature limit is very sensitive to approximations made in the derivation of the free energy, it would be worthwhile to extend the mathematical coarse-graining procedure of $\Gamma$-convergence to non-zero temperatures to obtain results that are more generally valid.

Based on this work, crystal plasticity models should be improved by extending the set of variables, such that the obtained free energy expression could be used. Particularly, the extension concerns including the densities for both positive and negative dislocations on each slip system, rather than only the GND-related quantities. The current derivation also suggests that, as the free energy is a strongly non-local functional, only the global version of the dissipation inequality should be used in the derivation of crystal plasticity models. It would be worthwhile to compare such a new crystal plasticity model with DDD simulations and, ultimately, experiments.

\section{Acknowledgments}

This work is part of the NWO Complexity programme, project CorFlux (nr. 10012310), which is financed by the Netherlands Organisation for Scientific Research (NWO).

\section{Appendix A. The mean field elastic energy}

In this section, the first three contributions of Eq. (2.20) are combined into a single expression in terms of $\boldsymbol{\varepsilon}_{\mathrm{mf}}$, as defined in Eq. (2.21). To this end, the first three contributions of Eq. (2.20) are rewritten using the definitions in Eqs. (2.6) and (2.12)

$$
\begin{aligned}
& \frac{1}{2} \int d V \varepsilon_{0}: \mathbb{C}: \boldsymbol{\varepsilon}_{0}+\frac{1}{2} \sum_{\boldsymbol{b}, \boldsymbol{b}^{\prime}} \int d A d A^{\prime} \rho_{\boldsymbol{b}}(\boldsymbol{s}) u_{\boldsymbol{b}, \boldsymbol{b}},\left(\boldsymbol{s}, \boldsymbol{s}^{\prime}\right) \rho_{\boldsymbol{b}},\left(\boldsymbol{s}^{\prime}\right)+\sum_{\boldsymbol{b}} \int d A \rho_{\boldsymbol{b}}(\boldsymbol{s}) V_{\boldsymbol{b}, 0}(\boldsymbol{s}) \\
& =\frac{1}{2} \int d V\left(\boldsymbol{\varepsilon}_{0}+\left(\nabla \times \int d V^{\prime} \mathrm{G}_{0}\left(\boldsymbol{r}, \boldsymbol{r}^{\prime}\right): \sum_{\boldsymbol{b}} \hat{\xi} \otimes \boldsymbol{b}_{\rho_{\boldsymbol{b}}}\left(\boldsymbol{r}_{2 D}^{\prime}\right)\right): \mathbb{S}\right): \mathbb{C}: \\
& :\left(\boldsymbol{\varepsilon}_{0}+\left(\nabla \times \int d V^{\prime} G_{0}\left(\boldsymbol{r}, \boldsymbol{r}^{\prime \prime}\right): \sum_{\boldsymbol{b}} \hat{\xi} \otimes \boldsymbol{b}_{\boldsymbol{b}}\left(\boldsymbol{r}_{2 D}^{\prime \prime}\right)\right): \mathbb{S}\right)
\end{aligned}
$$

To rewrite this equation, it should be used that the following combination is divergence free and symmetric:

$$
\mathbb{C}:\left(\boldsymbol{\varepsilon}_{0}+\left(\nabla \times \int d V^{\prime} \mathrm{G}_{0}\left(\boldsymbol{r}, \boldsymbol{r}^{\prime}\right): \sum_{\boldsymbol{b}} \hat{\boldsymbol{\xi}} \otimes \boldsymbol{b}_{\rho_{\boldsymbol{b}}}\left(\boldsymbol{r}_{2 D}^{\prime}\right)\right): \mathbb{S}\right) .
$$

These two properties will first be shown. First, the divergence free character of $\mathbb{C}: \boldsymbol{\varepsilon}_{0}$ follows immediately by Eq. (2.3), and $\nabla \times \int d V^{\prime} G_{0}\left(\boldsymbol{r}, \boldsymbol{r}^{\prime}\right): \sum_{\boldsymbol{b}} \hat{\xi} \otimes \boldsymbol{b}_{\rho_{\boldsymbol{b}}}\left(\boldsymbol{r}_{2 D}^{\prime}\right)$ is a rotation, and hence it is divergence free by definition.

Second, this combination is symmetric. For $\mathbb{C}: \boldsymbol{\varepsilon}_{0}$, this is immediately clear. For $\nabla \times \int d V^{\prime} G_{0}\left(\boldsymbol{r}, \boldsymbol{r}^{\prime}\right): \sum_{\boldsymbol{b}} \hat{\xi} \otimes \boldsymbol{b}_{\rho_{\boldsymbol{b}}}\left(\boldsymbol{r}_{2 D}^{\prime}\right)$, this is more subtle. As mentioned below Eq. (2.13), $\int d V^{\prime} G_{0}^{-1}\left(\boldsymbol{r}, \boldsymbol{r}^{\prime}\right): \Psi\left(\boldsymbol{r}^{\prime}\right)$ is infinite if $\nabla \times \boldsymbol{\Psi}(\boldsymbol{r})$ is not symmetric. Therefore, $\nabla \times G_{0}\left(\boldsymbol{r}, \boldsymbol{r}^{\prime}\right)$ should be symmetric in the first two indices. One can check that this is indeed the case for the infinite space solution for $\mathrm{G}_{0}$ in Eq. (2.14).

From these two properties, the following relationship can be derived for any arbitrary second order tensor field $\boldsymbol{h}(\boldsymbol{r})$ for which $\nabla \times \boldsymbol{h}(\boldsymbol{r})$ is symmetric: 


$$
\begin{aligned}
& \int d V\left(\boldsymbol{\varepsilon}_{0}+\left(\nabla \times \int d V^{\prime} \mathrm{G}_{0}\left(\boldsymbol{r}, \boldsymbol{r}^{\prime}\right): \sum_{\boldsymbol{b}} \hat{\xi} \otimes \boldsymbol{b}_{\boldsymbol{b}}\left(\boldsymbol{r}_{2 D}^{\prime}\right)\right): \mathbb{S}\right): \nabla \times \boldsymbol{h}(\boldsymbol{r}) \\
& =\int d V\left(\boldsymbol{\varepsilon}_{0}: \nabla \times \boldsymbol{h}(\boldsymbol{r})+\sum_{\boldsymbol{b}} \hat{\xi} \otimes \boldsymbol{b}_{\rho_{\boldsymbol{b}}}\left(\boldsymbol{r}_{2 D}^{\prime}\right): \boldsymbol{h}(\boldsymbol{r})\right) \\
& =\int d V\left(\boldsymbol{\varepsilon}_{0}: \nabla \times \boldsymbol{h}(\boldsymbol{r})-\nabla \times \boldsymbol{\beta}^{p}: \boldsymbol{h}(\boldsymbol{r})\right) \\
& =\int d V\left(\boldsymbol{\varepsilon}_{0}: \nabla \times \boldsymbol{h}(\boldsymbol{r})-\left(\boldsymbol{\beta}^{p}\right)^{s}: \nabla \times \boldsymbol{h}(\boldsymbol{r})\right)=\int d V \boldsymbol{\varepsilon}_{\mathrm{mf}}: \nabla \times \boldsymbol{h}(\boldsymbol{r})
\end{aligned}
$$

where in the first step the definition of $\mathrm{G}_{0}^{-1}$ in Eq. (2.13) is used, in the second the definition of $\boldsymbol{\beta}^{p}$ in Eq. (2.21), in the third Gauss' theorem and the symmetry of $\nabla \times \boldsymbol{h}(\boldsymbol{r})$ and in the fourth step the definition of $\boldsymbol{\varepsilon}_{\mathrm{mf}}$ in Eq. (2.21). By applying this identity twice in Eq. (A.1), it is found that

$$
\frac{1}{2} \int \mathrm{dV} \boldsymbol{\varepsilon}_{0}: \mathbb{C}: \boldsymbol{\varepsilon}_{0}+\frac{1}{2} \sum_{\boldsymbol{b}, \boldsymbol{b}^{\prime}} \int \mathrm{dAdA}^{\prime} \rho_{\boldsymbol{b}}(\boldsymbol{s}) u_{\boldsymbol{b}, \boldsymbol{b}^{\prime}}\left(\boldsymbol{s}, \boldsymbol{s}^{\prime}\right) \rho_{\boldsymbol{b}^{\prime}}\left(\boldsymbol{s}^{\prime}\right)+\sum_{\boldsymbol{b}} \int \mathrm{dA} \rho_{\boldsymbol{b}}(\boldsymbol{s}) V_{\boldsymbol{b}, 0}(\boldsymbol{s})=\frac{1}{2} \int \mathrm{dV} \boldsymbol{\varepsilon}_{\mathrm{mf}}: \mathbb{C}: \boldsymbol{\varepsilon}_{\mathrm{mf}} .
$$

This expression is used in Eq. (2.22).

\section{Appendix B. The Gradient correction in the LDA}

In this section, the gradient correction for the many body contribution is calculated using Eq. (4.2). To this end, the second derivative of the gradient correction with respect to the density profile is determined first:

$$
\begin{aligned}
\left.\frac{\delta^{2} \mathcal{F}_{m b}\left[\rho_{\text {tot }}\left(\boldsymbol{s}^{\prime}\right), T\right]}{\delta \rho_{\text {tot }}(\mathbf{0}) \delta \rho_{\text {tot }}(\boldsymbol{s})}\right|_{\rho_{\text {tot }}(\boldsymbol{s})=\rho_{\text {tot }}}= & -\operatorname{Tr}\left[\left(\operatorname{I} \delta\left(\boldsymbol{s}_{1}-\boldsymbol{s}_{2}\right)+\frac{1}{k_{B} T} \int d z d z^{\prime} \mathrm{G}_{0}\left(\boldsymbol{s}_{1}-\boldsymbol{s}_{2}, z-z^{\prime}\right):: \hat{\xi} \otimes \boldsymbol{b} \otimes \hat{\boldsymbol{\xi}} \otimes \boldsymbol{b} \rho_{\text {tot }}\right)\right. \\
& : \frac{1}{k_{B} T} \int d z d z^{\prime} \mathrm{G}_{0}\left(\boldsymbol{s}_{2}-\boldsymbol{s}_{3}, z-z^{\prime}\right): \hat{\boldsymbol{\xi}} \otimes \boldsymbol{b} \otimes \hat{\boldsymbol{\xi}} \otimes \boldsymbol{b}: \\
& :\left(\operatorname{I} \delta\left(\boldsymbol{s}_{3}-\boldsymbol{s}_{4}\right)+\frac{1}{k_{B} T} \int d z d z^{\prime} \mathrm{G}_{0}\left(\boldsymbol{s}_{3}-\boldsymbol{s}_{4}, z-z^{\prime}\right): \hat{\boldsymbol{\xi}} \otimes \boldsymbol{b} \otimes \hat{\boldsymbol{\xi}} \otimes \boldsymbol{b} \rho_{\text {tot }}\right) \\
& \left.:: \frac{1}{k_{B} T} \int d z d z^{\prime} \mathrm{G}_{0}\left(\boldsymbol{s}_{4}-\boldsymbol{s}_{1}, z-z^{\prime}\right): \hat{\boldsymbol{\xi}} \otimes \boldsymbol{b} \otimes \hat{\boldsymbol{\xi}} \otimes \boldsymbol{b}\right] \delta\left(\boldsymbol{s}_{1}\right) \delta\left(\boldsymbol{s}_{3}-\boldsymbol{s}\right) .
\end{aligned}
$$

The convolution on the RHS can be written as a product in Fourier space. Therefore, this expression reads, using the eigenvalues $\tilde{\lambda}(\boldsymbol{q})$ in Eq. (4.8),

$$
\left.\frac{\delta^{2} \mathcal{F}_{m b}\left[\rho_{S S D}(\boldsymbol{s}), T\right]}{\rho_{S S D}(\mathbf{0}) \rho_{S S D}(\boldsymbol{s})}\right|_{\text {PSSD }}=-\frac{1}{\rho_{S S D}^{2}} \frac{k_{B} T}{2} \frac{1}{(2 \pi)^{4}} \int d^{2} \boldsymbol{q} d^{2} \boldsymbol{q}^{\prime} e^{1(\boldsymbol{q} \prime-\boldsymbol{q}) \cdot \boldsymbol{s}} \frac{\tilde{\lambda}(\boldsymbol{q})}{1+\tilde{\lambda}(\boldsymbol{q})} \frac{\tilde{\lambda}\left(\boldsymbol{q}^{\prime}\right)}{1+\tilde{\lambda}\left(\boldsymbol{q}^{\prime}\right)} .
$$

The integrations over $\boldsymbol{q}$ and $\boldsymbol{q}^{\prime}$ can be performed independently. The function $j(\boldsymbol{s})$ is used as shorthand notation for the integral over $\mathbf{q}$ :

$$
j(\boldsymbol{s}) \equiv-\frac{1}{(2 \pi)^{2}} \int d^{2} \boldsymbol{q} e^{-1 \boldsymbol{q} \cdot \boldsymbol{s}} \frac{\tilde{\lambda}(\boldsymbol{q})}{1+\tilde{\lambda}(\boldsymbol{q})}=\frac{1}{\rho_{S S D}} \frac{1}{(2 \pi)^{2}} \int d^{2} \boldsymbol{q} e^{-1 \boldsymbol{q} \cdot \boldsymbol{s}} \frac{a q_{y}^{2}}{q^{4}+a q_{y}^{2}}
$$

where, as in the main text, $a$ is the shorthand notation for $a=\rho_{S S D} 2 \mu b^{2} L / k_{B} T(1-\nu)$. Eq. (B.2) can thus be written as

$$
\left.\frac{\delta^{2} \mathcal{F}_{m b}\left[\rho_{S S D}(\boldsymbol{s}), T\right]}{\delta \rho_{S S D}(\mathbf{0}) \delta \rho_{S S D}(\boldsymbol{s})}\right|_{\text {PSSD }}=-\frac{k_{B} T}{2} j(\boldsymbol{s}) j(-\boldsymbol{s})=-\frac{k_{B} T}{2} j(\boldsymbol{s})^{2},
$$

where the symmetry of $j(\boldsymbol{s})$ was used. Hence the matrix $\boldsymbol{F}_{2, m B}\left(\rho_{S S D}\right)$, as expressed in Eq. (4.2), reads

$$
\boldsymbol{F}_{2, m B}\left(\rho_{S S D}, T\right)=\frac{k_{B} T}{8} \int d A \boldsymbol{s} \otimes \boldsymbol{s} j(\boldsymbol{s})^{2} .
$$


This can be rewritten as follows:

$$
\begin{aligned}
\boldsymbol{F}_{2, m B}\left(\rho_{S S D}, T\right) & =\left.\frac{k_{B} T}{8} \int d A \boldsymbol{s} \otimes \boldsymbol{s} j(\boldsymbol{s})^{2} e^{1 \boldsymbol{q} \cdot \boldsymbol{s}}\right|_{\boldsymbol{q}=0} \\
& =-\left.\frac{k_{B} T}{8} \frac{\mathrm{d}}{\mathrm{d} \boldsymbol{q}} \otimes \frac{\mathrm{d}}{\mathrm{d} \boldsymbol{q}} \mathfrak{f}\left[j(\boldsymbol{s})^{2}\right](\boldsymbol{q})\right|_{\boldsymbol{q}=0} \\
& =-\left.\frac{k_{B} T}{8} \frac{\mathrm{d}}{\mathrm{d} \boldsymbol{q}} \otimes \frac{\mathrm{d}}{\mathrm{d} \boldsymbol{q}} \frac{1}{(2 \pi)^{2}} \int \mathrm{d} \boldsymbol{q}^{\prime} \tilde{j}\left(\boldsymbol{q}^{\prime}\right) \tilde{j}\left(\boldsymbol{q}-\boldsymbol{q}^{\prime}\right)\right|_{\boldsymbol{q}=0} \\
& =\frac{k_{B} T}{8} \frac{1}{(2 \pi)^{2}} \frac{a^{2}}{\rho_{S S D}^{2}} \int d \boldsymbol{q}^{\prime} \frac{\mathrm{d}}{\mathrm{d} \boldsymbol{q}^{\prime}} \tilde{j}\left(\boldsymbol{q}^{\prime}\right) \otimes \frac{\mathrm{d}}{\mathrm{d} \boldsymbol{q}^{\prime}} \tilde{j}\left(-\boldsymbol{q}^{\prime}\right) \\
& =\frac{k_{B} T}{8} \frac{1}{(2 \pi)^{2}} \frac{a^{2}}{\rho_{S S D}^{2}} \int d \boldsymbol{q}^{\prime}\left(\begin{array}{c}
q_{x}^{\prime} q_{y}^{\prime 2} q^{\prime 2} \\
-4 \frac{q_{y}^{\prime}\left(q_{x}^{\prime 4}-q_{y}^{\prime 4}\right)}{\left(q^{\prime 4}+a q_{y}^{\prime 2}\right)^{2}}
\end{array}\right) \otimes\left(\begin{array}{c}
q_{x}^{\prime} q_{y}^{\prime 2} q^{\prime 2} \\
-4 \frac{\left(q^{\prime 4}+a q_{y}^{\prime 2}\right)^{2}}{\left(q^{\prime}+a q_{y}^{\prime 2}\right)^{2}} \\
2 \frac{q_{y}^{\prime}\left(q_{x}^{\prime 4}-q_{y}^{\prime 4}\right)}{\left(q^{\prime 4}+a q_{y}^{\prime 2}\right)^{2}}
\end{array}\right),
\end{aligned}
$$

where in the third line, the inverse of the convolution theorem was used, and where for the last equality, the explicit form of $\tilde{j}(\boldsymbol{q})$ in Eq. (B.3) was used. These integrations can now be performed. Note that the $q_{x}$ derivative is antisymmetric in $q_{x}$, and the $q_{y}$ derivative is antisymmetric in $q_{y}$. Therefore, the off-diagonal terms in the matrix vanish. Using polar coordinates for the $\mathbf{q}^{\prime}$-integral, this yields

$$
\begin{aligned}
& \boldsymbol{F}_{2, m B}\left(\rho_{S S D}, T\right)=\frac{k_{B} T}{8} \frac{1}{(2 \pi)^{2}} \frac{4 a^{2}}{\rho_{S S D}^{2}} \\
& \int d q^{\prime} q^{\prime} \int_{0}^{2 \pi} d \phi \frac{q^{\prime 2} \cos ^{2} \phi}{\left(q^{\prime 2}+a \cos ^{2} \phi\right)^{4}}\left(\begin{array}{cc}
4 \cos ^{2} \phi \sin ^{2} \phi & 0 \\
0 & \left(\sin ^{4} \phi-\cos ^{4} \phi\right)^{2}
\end{array}\right) \\
& =\frac{k_{B} T}{8} \frac{1}{(2 \pi)^{2}} \frac{4 a^{2}}{\rho_{S S D}^{2}} \int d q^{\prime}\left(\begin{array}{cc}
\frac{\pi}{2\left(q^{\prime 2}+a\right)^{5 / 2}} & 0 \\
0 & \frac{\pi\left(4 q^{\prime 2}+a^{2}\right)}{8 q^{\prime 2}\left(q^{\prime 2}+a\right)^{7 / 2}}
\end{array}\right) \\
& =\frac{k_{B} T}{8} \frac{1}{(2 \pi)^{2}} \frac{4 a^{2}}{\rho_{S S D}^{2}}\left(\begin{array}{cc}
\frac{\pi}{3 a^{2}} & 0 \\
0 & -\frac{\pi}{3 a^{2}}+\frac{\pi}{8 a^{3 / 2} q_{0}}
\end{array}\right) \\
& =\frac{1}{24 \pi} \frac{k_{B} T}{\rho_{S S D}^{2}}\left(\begin{array}{lc}
1 & 0 \\
0 & -1+\frac{3 \sqrt{\rho_{S S D}}}{8 q_{0}} \sqrt{\frac{2 \mu b^{2} L}{k_{B} T(1-\nu)}}
\end{array}\right) \text {, }
\end{aligned}
$$

where $q_{0}$ is the lower bound of the $\boldsymbol{q}^{\prime}$-integral. The lower bound $q_{0}$ is inversely proportional to the largest length scale in the system, which is the system size $R$.

\section{Appendix C. Summation over glide planes}

In this appendix, the integrations over the $y$-coordinate and the summations over $n$ in Eq. (4.22) are performed. First, the summation in the first term can be evaluated:

$$
\int d y \sum_{n=-\infty}^{\infty} \delta(y-h n)=\sum_{n=-\infty}^{\infty} 1 \equiv N_{y},
$$

where the latter equality is the definition of $N_{y}$. The number $N_{y}$ can be interpreted as the number of glide planes. In the geometry considered here, this number is in principle infinite. However, it will be shown later that it is a common prefactor of all terms in Eq. (4.22).

Second, the summation in the second term can be evaluated using the explicit expression of the interaction potential in 
Eq. (2.14b):

$$
\begin{aligned}
& \int d y \int d y^{\prime} \sum_{n, n^{\prime} \in \mathbb{N}} \delta(y-h n) \delta\left(y^{\prime}-h n^{\prime}\right) u_{\text {edge }}\left(x-x^{\prime}, y-y^{\prime}\right)=\sum_{n=-\infty}^{\infty} \sum_{\Delta n=-\infty}^{\infty} u_{\text {edge }}\left(x-x^{\prime}, h \Delta n\right) \\
& =-N_{y} \frac{\mu b^{2} L}{2 \pi(1-\nu)} \sum_{\Delta n=-\infty}^{\infty}\left(\ln \left(\frac{\sqrt{\left(x-x^{\prime}\right)^{2}+h^{2} \Delta n^{2}}}{s_{0}}\right)+\frac{h^{2} \Delta n^{2}}{\left(x-x^{\prime}\right)^{2}+h^{2} \Delta n^{2}}\right) \\
& =-N_{y} \frac{\mu b^{2} L}{2 \pi(1-\nu)}\left(2 \sum_{\Delta n=1}^{\infty}\left(\ln \left(\frac{\sqrt{\left(x-x^{\prime}\right)^{2}+h^{2} \Delta n^{2}}}{h|\Delta n|}\right)+\ln \left(\frac{h|\Delta n|}{s_{0}}\right)\right)\right) \\
& +\ln \left(\frac{\left|x-x^{\prime}\right|}{s_{0}}\right)-N_{y} \frac{\mu b^{2} L}{2 \pi(1-\nu)} \sum_{\Delta n=-\infty}^{\infty}\left(1-\frac{\left(x-x^{\prime}\right)^{2}}{\left(x-x^{\prime}\right)^{2}+h^{2} \Delta n^{2}}\right),
\end{aligned}
$$

where in the first line $\Delta n$ is shorthand notation for $n-n^{\prime}$. To evaluate further, it is convenient to define $A$ by

$$
A \equiv 2 \sum_{\Delta n=1}^{\infty} \ln \left(\frac{h|\Delta n|}{s_{0}}\right),
$$

which is a constant independent of $x-x^{\prime}$. The summation does not converge as the number of glide planes is infinite. However, it will be shown later that the dislocation density profile is independent of $A$. Furthermore, the following identities can be used:

$$
\begin{gathered}
2 \sum_{\Delta n=1}^{\infty} \ln \left(\frac{\sqrt{\Delta x^{2}+h^{2} \Delta n^{2}}}{h|\Delta n|}\right)=\ln \left(\frac{h}{\pi \Delta x} \sinh \left(\pi \frac{\Delta x}{h}\right)\right) \\
\sum_{\Delta n=-\infty}^{\infty} \frac{\Delta x^{2}}{\Delta x^{2}+h^{2} \Delta n^{2}}=\pi \frac{\Delta x}{h} \operatorname{coth}\left(\pi \frac{\Delta x}{h}\right) .
\end{gathered}
$$

This finally yields

$$
\begin{gathered}
\int d y \int d y^{\prime} \sum_{n, n^{\prime} \in \mathbb{N}} \delta(y-h n) \delta\left(y^{\prime}-h n^{\prime}\right) u_{\text {edge }}\left(x-x^{\prime}, y-y^{\prime}\right) \\
=N_{y} \frac{\mu b^{2} L}{2 \pi(1-\nu)}\left(\pi \frac{\Delta x}{h} \operatorname{coth}\left(\pi \frac{\Delta x}{h}\right)-\ln \left(2 \sinh \left(\pi \frac{\Delta x}{h}\right)\right)\right) \\
-N_{y} \frac{\mu b^{2} L}{2 \pi(1-\nu)}\left(N_{y}+A+\ln \left(\frac{h}{2 \pi s_{0}}\right)\right),
\end{gathered}
$$

where the second term is a constant independent of $x-x^{\prime}$. An additive constant in the interaction potential has no physical meaning. Therefore, it will be neglected further on.

The interaction potential in the second line of Eq. (C.5) is equal to the interaction energy used in Scardia et al. (2014)], Geers et al. (2013)] and will be denoted by $u_{\text {wall }}(\Delta x)$ further on.

\section{References}

Bammann, D.J., 2001. A model of crystal plasticity containing a natural length scale. Mater. Sci. Eng. A 309-310 (July), 406-410.

Bargmann, S., Svendsen, B., 2012. Theoretical and algorithmic GND-based hardening in single crystals. Int. J. Multisc. Comput. 10 (6), $551-565$.

Baskaran, R., Akarapu, S., Mesarovic, S.D., Zbib, H.M., 2010. Energies and distributions of dislocations in stacked pile-ups. Int. J. Solids Struct. 47 (May 9), $1144-1153$.

Bayley, C.J., Brekelmans, W.A.M., Geers, M.G.D., 2007. A three-dimensional dislocation field crystal plasticity approach applied to miniaturized structures. Philos. Mag. 87 (March 8-9), 1361-1378.

Burakovsky, L., Preston, D., Silbar, R., 2000. Melting as a dislocation-mediated phase transition. Phys. Rev. B 61 (June (22)), 15011-15018.

Carlson, D., 1966. On the completeness of the Beltrami stress functions in continuum mechanics. J. Math. Anal. Appl. 15 (August (2)), $311-315$.

Chaikin, P., Lubensky, T., 1995. Principles of Condensed Matter Physics. Cambridge University Press, Cambridge, United Kingdom.

Ekh, M., Grymer, M., Runesson, K., Svedberg, T., 2007. Gradient crystal plasticity as part of the computational modelling of polycrystals. Int. J. Numer. Methods Eng. 72, 197-220.

Ertürk, İ., van Dommelen, J.A.W., Geers, M.G.D., 2009. Energetic dislocation interactions and thermodynamical aspects of strain gradient crystal plasticity theories. J. Mech. Phys. Solids 57 (November (11)), 1801-1814.

Evans, R., 1979. The nature of the liquid-vapour interface and other topics in the statistical mechanics of non-uniform, classical fluids. Adv. Phys. 28 (2), $143-200$.

Evers, L., 2004. Non-local crystal plasticity model with intrinsic SSD and GND effects. J. Mech. Phys. Solids 52 (October (10)), $2379-2401$.

Evers, L., Brekelmans, W., Geers, M.G.D., 2004. Scale dependent crystal plasticity framework with dislocation density and grain boundary effects. Int. J.

Solids Struct. 41 (September (18-19)), 5209-5230. 
Geers, M.G.D., Peerlings, R.H.J., Peletier, M.A., Scardia, L., 2013. Asymptotic behaviour of a pile-up of infinite Walls of Edge Dislocations. Arch. Rational Mech. Anal. 209, 495-539.

Groma, I., 1997. Link between the microscopic and mesoscopic length-scale description of the collective behavior of dislocations. Phys. Rev. B 56 (10), $5807-5813$.

Groma, I., Balogh, P., 1999. Investigation of dislocation pattern formation in a two-dimensional self-consistent field approximation. Acta Mater. 47 (October (13)), 3647-3654.

Groma, I., Györgyi, G., Ispánovity, P., 2010. Variational approach in dislocation theory. Philos. Mag. 90 (September (27-28)), 3679-3695.

Groma, I., Györgyi, G., Kocsis, B., 2006. Debye screening of dislocations. Phys. Rev. Lett. 96 (April (16)), 165503.

Groma, I., Györgyi, G., Kocsis, B., 2007. Dynamics of coarse grained dislocation densities from an effective free energy. Philos. Mag. 87 (March (8-9)), 1185-1199.

Groma, I., Vandrus, Z., Ispánovity, P., 2014. Scale-free phase field theory of dislocations. ArXiv (1404.6344v1 [cond-mat.mtrl-sci]).

Gurtin, M.E., 2010. A finite-deformation, gradient theory of single-crystal plasticity with free energy dependent on the accumulation of geometrically necessary dislocations. Int. J. Plast. 26 (August (8)), 1073-1096.

Gurtin, M.E., 2000. On the plasticity of single crystals: free energy, microforces, plastic-strain gradients. J. Mech. Phys. Solids 48 (May (5)), 989-1036.

Gurtin, M.E., 2008. A finite-deformation, gradient theory of single-crystal plasticity with free energy dependent on densities of geometrically necessary dislocations. Int. J. Plast. 24 (April (4)), 702-725.

Gurtin, M.E., Anand, L., 2005. A theory of strain-gradient plasticity for isotropic, plastically irrotational materials. Part I: small deformations. J. Mech. Phys. Solids 53 (July (7)), 1624-1649.

Gurtin, M.E., Anand, L., Lele, S.P., 2007. Gradient single-crystal plasticity with free energy dependent on dislocation densities. J. Mech. Phys. Solids 55 (September (9)), 1853-1878.

Hirth, J.P., Lothe, J., 1982. Theory of Dislocations, second edition. Krieger Publishing Company, Malabar, Florida.

Hochrainer, T., Sandfeld, S., Zaiser, M., Gumbsch, P., 2014. Continuum dislocation dynamics: towards a physical theory of crystal plasticity. J. Mech. Phys. Solids 63, 167-178.

Ispánovity, P., Groma, I., Györgyi, G., Csikor, F., Weygand, D., 2010. Submicron plasticity: yield stress, dislocation avalanches, and velocity distribution. Phys. Rev. Lett. 105 (August (8)).

Kleinert, H., 1989. Gauge Fields in Condensed Matter. World Scientific Publishing Co. Pte. Ltd, Singapore.

Klusemann, B., Bargmann, S., Svendsen, B., 2012. Two models for gradient inelasticity based on non-convex energy. Comput. Mater. Sci. 64 (November).

Kooiman, M., Hütter, M., Geers, M.G.D., 2014, Collective behaviour of dislocations in a finite medium. J. Stat. Mech. Theory Exp. P04028, http://dx.doi.org/10. 1088/1742-5468/2014/04/P04028.

Kosterlitz, J.M., Thouless, D.J., 1973. Ordering, metastability and phase transitions in two-dimensional systems. J. Phys. C 6.

Kuroda, M., Tvergaard, V., 2008. A finite deformation theory of higher-order gradient crystal plasticity. J. Mech. Phys. Solids 56 (August (8)), $2573-2584$.

Landau, L.D., Lifshitz, E.M., 1975. The Classical Theory of Fields. Elsevier, Oxford, United Kingdom, fourth rev edition.

Limkumnerd, S., Van der Giessen, E., 2008. Statistical approach to dislocation dynamics: from dislocation correlations to a multiple-slip continuum theory of plasticity. Phys. Rev. B 77 (May (18)), 184111.

Mesarovic, S.D., 2005. Energy, configurational forces and characteristic lengths associated with the continuum description of geometrically necessary dislocations. Int. J. Plast. 21 (October (10), 1855-1889.

Mesarovic, S.D., Baskaran, R., Panchenko, A., 2010. Thermodynamic coarsening of dislocation mechanics and the size-dependent continuum crystal plasticity. J. Mech. Phys. Solids 58 (March (3)), 311-329.

Mizushima, S., 1960. Dislocation model of liquid structure. J. Phys. Soc. Jpn. 15 (1), 70-77.

Nelson, D.R., 1978. Study of melting in two dimensions. Phys. Rev. B 18 (5).

Nelson, D.R., Halperin, B.I., 1979. Dislocation-mediated melting in two dimensions. Phys. Rev. B 19 (5).

Ninomiya, T., 1978. Theory of melting, dislocation model. I. J. Phys. Soc. Jpn. 44 (1).

Panyukov, S., Rabin, Y., 1999. Statistical physics of interacting dislocation loops and their effect on the elastic moduli of isotropic solids. Phys. Rev. B 59 (21), 13657-13671.

Raabe, D., Roters, F., Barlat, F., Chen, L., 2004. Continuum Scale Simulation of Engineering Materials. Wiley, Weinheim.

Scardia, L., Peerlings, R., Peletier, M., Geers, M.G.D., 2014. Mechanics of dislocation pile-ups: a unification of scaling regimes. J. Mech. Phys. Solids 70 (October), 42-61.

Smallman, R., Ngan, A., 2011. Physical Metallurgy and Advanced Materials. Elsevier Science, Oxford, United Kingdom.

Svendsen, B., 2002. Continuum thermodynamic models for crystal plasticity including the e ects of geometrically-necessary dislocations. J. Mech. Phys. Solids 50, 1297-1329.

Svendsen, B., Bargmann, S., 2010. On the continuum thermodynamic rate variational formulation of models for extended crystal plasticity at large deformation. J. Mech. Phys. Solids 58 (September (9)), 1253-1271.

Van der Giessen, E., Needleman, A., 1995. Discrete dislocation plasticity: a simple planar model. Model. Simul. Mater. Sci. Eng. 3, $689-735$.

Yamamoto, T., Izuyama, T., 1988. Statistical mechanics of the dislocation system in a crystal. J. Phys. Soc. Jpn. 57 (11), $3742-3752$. 